\title{
Reaction of Allenyl Esters with Sodium Azide: An Efficient Synthesis of E-Vinyl Azides and Polysubstituted Pyrroles
}

\author{
Xian Huang* a, b, Ruwei Shen ${ }^{\mathrm{a}}$ and Tiexin Zhang ${ }^{\mathrm{a}}$
}

a. Department of Chemistry, Zhejiang University (Xixi Campus), Hangzhou 310028, P. R. China

b. State Key Laboratory of Organometallic Chemistry, Shanghai Institute of Organic Chemistry, Chinese Academy of Sciences, Shanghai 200032, P. R. China

huangx@mail.hz.zj.cn

\section{Supporting Information \\ List of contents}

Experiment procedures.....................................S2/S3

Characterization data of key compounds.....................S4-S13

${ }^{1} \mathrm{H}$ NMR and ${ }^{13} \mathrm{C}$ NMR spectra of key compounds................S14-S39

NOESY spectra for compounds $\boldsymbol{E}$-2d, Z-3d and $\boldsymbol{E}$-3d...........S40/S41 


\section{Experiment procedures}

General. All ${ }^{1} \mathrm{H}$ NMR and ${ }^{13} \mathrm{C}$ NMR spectra were measured in $\mathrm{CDCl}_{3}$ with TMS as the internal standard. Chemical shifts are expressed in ppm and $\mathbf{J}$ values are given in Hz. Reagents in liquid state were purified by distillation and Reagents in solid state used as received without further purification. Petroleum ether refers to the fraction with boiling point in the range $60-90^{\circ} \mathrm{C} .1$, 2-allenyl esters $\mathbf{1}$ and 1' were prepared according to the known method by treatment of the acid chlorides with the corresponding ethyl 2-(triphenylphoranylidene) propionate. ${ }^{1}$

NOTE: there exist potential dangers of using azides on large scale, and the workup involving quenching with ammonium chloride described in this paper can result in ammonium azide or hydrazoic acid which may cause explosion. No hazard evaluation was conducted on the reaction. Therefore special caution should be taken before carrying on the reaction on large scale.

The reaction of 1,2-allenyl esters with $\mathrm{NaN}_{3}$ at room temperature. Preparation of 2 or 3. Typical procedure. To a solution of $\mathrm{NaN}_{3}(1 \mathrm{mmol})$ in $t-\mathrm{BuOH} / \mathrm{H}_{2} \mathrm{O}(\mathrm{v} / \mathrm{v}=4: 1$, $2 \mathrm{ml})$, ethyl 4-phenylbuta-2,3-dienoate $(0.5 \mathrm{mmol})$ was added at room temperature with stirring. After the reaction was complete $(30 \mathrm{~min})$, the reaction was quenched with saturated $\mathrm{NH}_{4} \mathrm{Cl}$ and extracted with EtOAc $(3 \times .10 \mathrm{~mL})$. The organic phase was 
washed with saturated brine and dried over $\mathrm{MgSO}_{4}$. After filtration and removal of the solvent in vacuo, the residues were purified with flash chromatography (silica/ Petroleum ether - ethyl acetate $20: 1 \mathrm{v} / \mathrm{v})$ to afford $110 \mathrm{mg}(95 \%)$ of 2a. NOTE: $\boldsymbol{E}-2$ and Z-2 were inseparable and obtained as mixture; $\boldsymbol{E}$-3 and $\mathbf{Z}-\mathbf{3}$ were obtained respectively.

The reaction of 1-allylic 1,2-allenyl esters with $\mathrm{NaN}_{3}$ at $65^{\circ} \mathrm{C}$. Preparation of 5 . Typical procedure.

A solution of $3 \mathrm{mmol}$ of $\mathrm{NaN}_{3}, 1 \mathrm{mmol}$ ethyl 2-vinylidenepent-4-enoate in $3 \mathrm{~mL}$ of $t$-BuOH was heated to $65{ }^{\circ} \mathrm{C}$ with stirring. After the reaction was complete (monitored by TLC), the reaction was quenched with saturated $\mathrm{NH}_{4} \mathrm{Cl}$ and extracted with EtOAc $(3 \times 15 \mathrm{~mL})$. The organic phase was washed with saturated brine and dried over $\mathrm{MgSO}_{4}$. After filtration and removal of the solvent in vacuo, the residues were purified with flash chromatography (silica/ Petroleum ether - ethyl acetate $6: 1 \mathrm{v} / \mathrm{v}$ ) to afford $152 \mathrm{mg}(91 \%)$ of $5 a$.

\section{Procedure for thermal reaction of $E$-3c to afford 5 a.}

A solution of $0.5 \mathrm{mmol} \boldsymbol{E}$-3c in $1 \mathrm{~mL} t$-BuOH was heated to $80{ }^{\circ} \mathrm{C}$ with stiring. After the reaction was complete ( 3 hours, monitored by TLC), the mixture was directly purified with flash chromatography (silica/ Petroleum ether - ethyl acetate $6: 1 \mathrm{v} / \mathrm{v}$ ) to afford $79 \mathrm{mg}(95 \%)$ of $5 a$. 


\section{Characterization data of key compounds}

\section{(E)-ethyl 3-azido-4-phenylbut-3-enoate ( $E$-2a)}

oil. ${ }^{1} \mathrm{H}$ NMR (400 MHz, $\mathrm{CDCl}_{3}$ ): 7.36-7.23 (m, 5H), 6.41 (s, 1H), 4.24 (q, J=7.6 Hz, 2H), $3.30(\mathrm{~s}, 2 \mathrm{H}), 1.30(\mathrm{t}, J=7.6 \mathrm{~Hz}, 3 \mathrm{H}) .{ }^{13} \mathrm{C} \mathrm{NMR}\left(100 \mathrm{MHz}, \mathrm{CDCl}_{3}\right): 169.5,135.0$, 133.0, 128.5, 128.3, 127.2, 118.6, 61.4, 35.9, 14.1. IR (neat): 2982, 2100, 1737, 1666, $1609 \mathrm{~cm}^{-1}$. MS: m/z (\%) $231\left(\mathrm{M}^{+}, 4\right), 203$ (8), 105 (100). Anal. Calcd for $\mathrm{C}_{12} \mathrm{H}_{13} \mathrm{~N}_{3} \mathrm{O}_{2}$ (231.25): C 62.33, H 5.67, N 18.17. Found: C 62.41, H 5.56, N 18.06.

\section{(E)-ethyl 3-azidopent-3-enoate $(E-2 b)$}

oil. ${ }^{1} \mathrm{H}$ NMR (400 MHz, $\mathrm{CDCl}_{3}$ ): 5.34 (q, $\left.J=7.2 \mathrm{~Hz}, 1 \mathrm{H}\right), 4.15$ (q, $J=7.2 \mathrm{~Hz}, 2 \mathrm{H}$ ), $3.09(\mathrm{~s}, 2 \mathrm{H}), 1.69(\mathrm{~d}, J=7.2 \mathrm{~Hz}, 3 \mathrm{H}), 1.25(\mathrm{t}, J=7.2 \mathrm{~Hz}, 3 \mathrm{H}) .{ }^{13} \mathrm{C}$ NMR $(100 \mathrm{MHz}$, $\left.\mathrm{CDCl}_{3}\right): 169.3,131.0,112.8,61.1,34.4,14.0,12.7$. IR neat): 2926, 2118, 1740, 1655 $\mathrm{cm}^{-1}$. MS: m/z (\%) $169\left(\mathrm{M}^{+}, 3\right), 141$ (16), 43 (100). Anal. Calcd for $\mathrm{C}_{7} \mathrm{H}_{11} \mathrm{~N}_{3} \mathrm{O}_{2}$ (169.18): C 49.70, H 6.55, N 24.84. Found: C 49.59, H 6.46, N 25.01.

\section{(E)-ethyl 3-azidohex-3-enoate $(E-2 c)$}

oil. ${ }^{1} \mathrm{H}$ NMR (400 MHz, $\mathrm{CDCl}_{3}$ ): 5.30 (t, $\left.J=7.6 \mathrm{~Hz}, 1 \mathrm{H}\right), 4.18$ (q, $\left.J=7.2 \mathrm{~Hz}, 2 \mathrm{H}\right)$, $3.10(\mathrm{~s}, 2 \mathrm{H}), 2.15-2.08(\mathrm{~m}, 2 \mathrm{H}), 1.28(\mathrm{t}, J=7.2 \mathrm{~Hz}, 3 \mathrm{H}), 1.01(\mathrm{t}, J=7.6 \mathrm{~Hz}, 3 \mathrm{H}) .{ }^{13} \mathrm{C}$ NMR (100 MHz, $\left.\mathrm{CDCl}_{3}\right): 169.4,130.1,120.1,61.2,34.8,21.0,14.2,14.1$. IR (neat): 2970, 2100, 1740, $1658 \mathrm{~cm}^{-1}$. MS: m/z (\%) $183\left(\mathrm{M}^{+}, 2\right), 155$ (18), 41(100). Anal. 
Calcd for $\mathrm{C}_{8} \mathrm{H}_{13} \mathrm{~N}_{3} \mathrm{O}_{2}$ (183.21): C 52.45, H 7.15, N 22.94. Found: C 52.51, H 7.28, N 22.76 .

\section{(E)-ethyl 3-azido-4-(naphthalen-8-yl)but-3-enoate (E-2d)}

oil. ${ }^{1} \mathrm{H}$ NMR (400 MHz, $\left.\mathrm{CDCl}_{3}\right)$ : 7.97-7.80 (m, 3H), 7.53-7.40 (m, 4H), 6.85 (s, 1H), $4.20(\mathrm{q}, J=7.2 \mathrm{~Hz}, 2 \mathrm{H}), 3.23(\mathrm{~s}, 2 \mathrm{H}), 1.28(\mathrm{t}, J=7.2 \mathrm{~Hz}, 3 \mathrm{H}) .{ }^{13} \mathrm{C}$ NMR $(100 \mathrm{MHz}$, $\left.\mathrm{CDCl}_{3}\right): 169.5,134.5,133.5,132.1,131.9,128.4,128.1,126.7,126.3,126.1,125.4$, 124.6, 116.5, 61.3, 35.9, 14.0. IR (neat): 2981, 2103, 1736, $1646 \mathrm{~cm}^{-1} . \mathrm{MS}: \mathrm{m} / \mathrm{z}(\%)$ $281\left(\mathrm{M}^{+}, 1\right), 253$ (36), 180 (100). Anal. Calcd for $\mathrm{C}_{16} \mathrm{H}_{15} \mathrm{~N}_{3} \mathrm{O}_{2}$ (281.31): C 68.31, H 5.37, N 14.94. Found: C 68.18, H 5.31, N 15.06.

\section{(E)-ethyl 3-azidooct-3-enoate $(E-2 e)$}

oil. ${ }^{1} \mathrm{H}$ NMR (400 MHz, $\left.\mathrm{CDCl}_{3}\right): 5.25$ (t, $\left.J=7.6 \mathrm{~Hz}, 1 \mathrm{H}\right), 4.12(\mathrm{q}, J=7.2 \mathrm{~Hz}, 2 \mathrm{H})$, $3.06(\mathrm{~s}, 2 \mathrm{H}), 2.07-2.01(\mathrm{~m}, 2 \mathrm{H}), 1.34-1.27(\mathrm{~m}, 4 \mathrm{H}), 1.23(\mathrm{t}, J=7.2 \mathrm{~Hz}, 3 \mathrm{H}), 0.85(\mathrm{t}, J$ $=6.8 \mathrm{~Hz}, 3 \mathrm{H}) .{ }^{13} \mathrm{C} \mathrm{NMR}\left(100 \mathrm{MHz}, \mathrm{CDCl}_{3}\right): 169.3,130.3,118.5,61.0,34.6,31.6$, 27.1, 22.0, 13.9, 13.7. IR (neat): 2959, 2099, 1741, $1656 \mathrm{~cm}^{-1} . \mathrm{MS}: \mathrm{m} / \mathrm{z}(\%) 212$ $\left(\mathrm{M}^{+}+1,1\right), 180$ (76), 41 (100). Anal. Calcd for $\mathrm{C}_{10} \mathrm{H}_{17} \mathrm{~N}_{3} \mathrm{O}_{2}$ (211.26): C 56.85, H 8.11, N 19.89. Found: C 57.03, H 7.98, N 19.78.

\section{(E)-ethyl 3-azidoundec-3-enoate (E-2f)}

oil. ${ }^{1} \mathrm{H}$ NMR (400 MHz, $\left.\mathrm{CDCl}_{3}\right): 5.27$ (t, $\left.J=7.2 \mathrm{~Hz}, 1 \mathrm{H}\right), 4.14$ (q, $\left.J=7.2 \mathrm{~Hz}, 2 \mathrm{H}\right)$, 
$3.08(\mathrm{~s}, 2 \mathrm{H}), 2.08-2.02(\mathrm{~m}, 2 \mathrm{H}), 1.37-1.32(\mathrm{~m}, 2 \mathrm{H}), 1.26-1.23(\mathrm{~m}, 11 \mathrm{H}), 0.85(\mathrm{t}, J=$ $6.8 \mathrm{~Hz}, 3 \mathrm{H}) .{ }^{13} \mathrm{C} \mathrm{NMR}\left(100 \mathrm{MHz}, \mathrm{CDCl}_{3}\right): 169.3,130.3,118.7,61.1,34.7,31.7,29.5$, 29.0, 28.9, 27.5, 22.5, 13.99, 13.96. IR (neat): 2928, 2102, 1742, $1657 \mathrm{~cm}^{-1} . \mathrm{MS}: \mathrm{m} / \mathrm{z}$ (\%) $254\left(\mathrm{M}^{+}+1,2\right), 226$ (100). Anal. Calcd for $\mathrm{C}_{13} \mathrm{H}_{23} \mathrm{~N}_{3} \mathrm{O}_{2}$ (253.34): C 61.63, H 9.15, N 16.59. Found: C 61.71, H 9.31, N 16.46.

\section{(E)-ethyl 3-azidobut-2-enoate (E-3a)}

oil. ${ }^{1} \mathrm{H}$ NMR (400 MHz, $\left.\mathrm{CDCl}_{3}\right): 5.50$ (s, 1H), 4.14 (q, J=6.8 Hz, 2H), 2.32 (s, 3H), $1.26(\mathrm{t}, J=6.8 \mathrm{~Hz}, 3 \mathrm{H}) .{ }^{13} \mathrm{C} \mathrm{NMR}\left(100 \mathrm{MHz}, \mathrm{CDCl}_{3}\right)$ : 166.2, 154.6, 105.1, 59.9, 16.2, 14.2. IR (neat): 2983, 2098, 1713, $1628 \mathrm{~cm}^{-1}$. MS: m/z (\%) $155\left(\mathrm{M}^{+}, 6\right), 127(9), 43$ (100). Anal. Calcd for $\mathrm{C}_{6} \mathrm{H}_{9} \mathrm{~N}_{3} \mathrm{O}_{2}$ (155.15): C 46.45, H 5.85, N 27.08. Found: C 46.61, H 5.78, N 26.96.

\section{(E)-ethyl 3-azido-2-methylbut-2-enoate $(E-3 b)$}

oil. ${ }^{1} \mathrm{H}$ NMR (400 MHz, $\mathrm{CDCl}_{3}$ ): 4.17 (q, $\left.J=7.2 \mathrm{~Hz}, 2 \mathrm{H}\right), 2.4(\mathrm{~d}, J=1.4 \mathrm{~Hz}, 3 \mathrm{H}), 1.8$ $(\mathrm{d}, J=1.4 \mathrm{~Hz}, 3 \mathrm{H}), 1.28(\mathrm{t}, J=7.2 \mathrm{~Hz}, 3 \mathrm{H}) .{ }^{13} \mathrm{C} \mathrm{NMR}\left(100 \mathrm{MHz}, \mathrm{CDCl}_{3}\right): 168.0$, 143.9, 114.5, 60.3, 16.0, 14.2, 13.9. IR (neat): 2118, 1724, $1665 \mathrm{~cm}^{-1} . \mathrm{MS}: \mathrm{m} / \mathrm{z}(\%)$ $169\left(\mathrm{M}^{+}, 16\right), 141$ (18), 43 (100). Anal. Calcd for $\mathrm{C}_{7} \mathrm{H}_{11} \mathrm{~N}_{3} \mathrm{O}_{2}$ (169.18): C 49.70, $\mathrm{H}$ 6.55, N 24.84. Found: C 49.63, H 6.69, N 25.01.

\section{(E)-ethyl 2-(1-azidoethylidene)pent-4-enoate $(E-3 c)$}


oil. ${ }^{1} \mathrm{H}$ NMR (400 MHz, $\left.\mathrm{CDCl}_{3}\right): 5.81-5.71(\mathrm{~m}, 1 \mathrm{H}), 4.96-5.03$ (m, 2H), 4.17 (q, $J=$ $7.2 \mathrm{~Hz}, 2 \mathrm{H}), 3.09$ (d, $J=6.0 \mathrm{~Hz}, 2 \mathrm{H}), 2.43(\mathrm{~s}, 3 \mathrm{H}), 1.27$ (t, $J=7.2 \mathrm{~Hz}, 3 \mathrm{H}) .{ }^{13} \mathrm{C} \mathrm{NMR}$ (100 MHz, $\left.\mathrm{CDCl}_{3}\right): 167.4,144.7,135.6,117.0,115.1,60.3,32.2,16.1,14.1$. IR (neat): 2982, 2119, 1704, 1619, $1280 \mathrm{~cm}^{-1} . \mathrm{MS}: \mathrm{m} / \mathrm{z}(\%) 195\left(\mathrm{M}^{+}, 1\right), 167$ (15), 43 (100). Anal. Calcd for $\mathrm{C}_{9} \mathrm{H}_{13} \mathrm{~N}_{3} \mathrm{O}_{2}$ (195.22): C 55.37, H 6.71, N 21.52. Found: C 55.45, H 6.85, N 21.66.

\section{(E)-ethyl 3-azido-2-benzylbut-2-enoate (E-3d)}

oil. ${ }^{1} \mathrm{H}$ NMR (400 MHz, $\left.\mathrm{CDCl}_{3}\right): 7.25-7.15$ (m, 5H), 4.11 (q, $\left.J=7.2 \mathrm{~Hz}, 2 \mathrm{H}\right), 3.71$ (s, 2H), 2.48 (s, 3H), $1.19(\mathrm{t}, J=7.2 \mathrm{~Hz}, 3 \mathrm{H}) .{ }^{13} \mathrm{C} \mathrm{NMR}\left(100 \mathrm{MHz}, \mathrm{CDCl}_{3}\right): 167.4,145.1$, 140.2, 128.4, 128.1, 125.9, 118.3, 60.4, 33.6, 16.2, 14.1. IR (neat): 2982, 2118, 1705, 1618, $1280 \mathrm{~cm}^{-1}$. MS: m/z (\%) $246\left(\mathrm{M}^{+}+1,4\right), 245\left(\mathrm{M}^{+}, 1\right), 218$ (100). Anal. Calcd for $\mathrm{C}_{13} \mathrm{H}_{15} \mathrm{~N}_{3} \mathrm{O}_{2}$ (245.28): C 63.66, H 6.16, N 17.13. Found: C 63.53, H 6.25, N 17.29 .

\section{(Z)-ethyl 3-azido-2-benzylbut-2-enoate (Z-3d)}

oil. ${ }^{1} \mathrm{H}$ NMR (400 MHz, $\left.\mathrm{CDCl}_{3}\right)$ : 7.27-7.15 (m, 5H), 4.14 (q, $\left.J=7.2 \mathrm{~Hz}, 2 \mathrm{H}\right), 3.68$ (s, 2H), 2.13 (s, 3H), 1.19 (t, $J=7.2 \mathrm{~Hz}, 3 \mathrm{H}) .{ }^{13} \mathrm{C}$ NMR (100 MHz, $\left.\mathrm{CDCl}_{3}\right):$ 166.9, 139.7, 138.9, 128.5, 128.0, 126.3, 117.8, 60.6, 35.2, 15.9, 14.1. IR (neat): 2968, 2101, 1715, 1619, $1278 \mathrm{~cm}^{-1}$. MS: m/z (\%) $246\left(\mathrm{M}^{+}+1,4\right), 245\left(\mathrm{M}^{+}, 1\right), 218(100)$. 
(E)-ethyl 2-(1-azidoethylidene)-4-methylpent-4-enoate (E-3e)

oil. ${ }^{1} \mathrm{H}$ NMR (400 MHz, $\mathrm{CDCl}_{3}$ ): 4.68 (s, 1H), 4.56 (s, 1H), 4.15 (q, J=7.2 Hz, 2H), $3.05(\mathrm{~s}, 2 \mathrm{H}), 2.43(\mathrm{~s}, 3 \mathrm{H}), 1.70(\mathrm{~s}, 3 \mathrm{H}), 1.25(\mathrm{t}, J=7.2 \mathrm{~Hz}, 3 \mathrm{H}) .{ }^{13} \mathrm{C}$ NMR $(100 \mathrm{MHz}$ $\left.\mathrm{CDCl}_{3}\right): 167.6,144.4,143.5,117.5,110.0,60.3,35.7,22.7,16.0,14.1$. IR (neat): 2980, 2118, 1705, 1621, $1288 \mathrm{~cm}^{-1} . \mathrm{MS}: \mathrm{m} / \mathrm{z}(\%) 209\left(\mathrm{M}^{+}, 2\right), 181$ (19), 43 (100). Anal. Calcd for $\mathrm{C}_{10} \mathrm{H}_{15} \mathrm{~N}_{3} \mathrm{O}_{2}$ (209.24): C 57.40, H 7.23, N 20.08. Found: C 57.53, H 7.35, N 19.93.

(E)-ethyl 2-(1-(4-phenyl-1H-1,2,3-triazol-1-yl)ethylidene)pent-4-enoate (4a) oil. ${ }^{1} \mathrm{H}$ NMR (400 MHz, $\left.\mathrm{CDCl}_{3}\right)$ : 7.88 (s, 1H), 7.86-7.84 (m, 2H), 7.47-7.43 (m, 2H), 7.38-7.36 (m, 1H), 5.88-5.78 (m, 1H), 5.12-5.04 (m, 2H), 4.32 (q, J = 7.2 Hz, 2H), 2.99-2.97 (m, 2H), $2.54(\mathrm{~s}, 3 \mathrm{H}), 1.35(\mathrm{t}, J=7.2 \mathrm{~Hz}, 3 \mathrm{H}) .{ }^{13} \mathrm{C}$ NMR (100 MHz, $\left.\mathrm{CDCl}_{3}\right): 167.2,147.3,139.8,134.3,129.9,128.9,128.6,128.4,125.7,119.9,116.9$ 61.4, 33.8, 21.2, 14.1. IR (neat): 3137, 2924, 1722, $1647 \mathrm{~cm}^{-1} . \mathrm{MS}: \mathrm{m} / \mathrm{z}(\%) 298$ $\left(\mathrm{M}^{+}+1,18\right), 240$ (77), 117 (100). Anal. Calcd for $\mathrm{C}_{17} \mathrm{H}_{19} \mathrm{~N}_{3} \mathrm{O}_{2}$ (297.35): C 68.67, $\mathrm{H}$ 6.44, N 14.13. Found: C 68.61, H 5.36, N 14.25.

(E)-ethyl 3-(4-phenyl-1H-1,2,3-triazol-1-yl)undec-3-enoate (4b)

oil. ${ }^{1} \mathrm{H}$ NMR (400 MHz, $\left.\mathrm{CDCl}_{3}\right)$ : 8.02 (s, 1H), 7.85-7.83 (m, 2H), 7.42-7.38 (m, 2H), 7.33-7.30 (m, 1H), $6.09(\mathrm{t}, J=7.6 \mathrm{~Hz}, 1 \mathrm{H}), 4.12(\mathrm{q}, J=7.2 \mathrm{~Hz}, 2 \mathrm{H}), 3.82(\mathrm{~s}, 2 \mathrm{H})$, 
2.26-2.21 (m, 2H), 1.52-1.45 (m, 2H), 1.34-1.25 (m, 8H), $1.20(\mathrm{t}, J=7.2 \mathrm{~Hz}, 3 \mathrm{H})$, $0.88(\mathrm{t}, J=6.8 \mathrm{~Hz}, 3 \mathrm{H}) .{ }^{13} \mathrm{C} \mathrm{NMR}\left(100 \mathrm{MHz}, \mathrm{CDCl}_{3}\right): 169.2,147.3,130.3,129.8$, $128.7,128.1,125.8,125.6,117.8,61.2,34.5,31.6,29.1,29.0,28.9,27.4,22.5,14.0$, 13.9. IR (neat): $3141,2928,1741,1460 \mathrm{~cm}^{-1} . \mathrm{MS}: \mathrm{m} / \mathrm{z}(\%) 356\left(\mathrm{M}^{+}+1,7\right), 327$ (39), 81 (100). Anal. Calcd for $\mathrm{C}_{21} \mathrm{H}_{29} \mathrm{~N}_{3} \mathrm{O}_{2}$ (355.47): C 70.95, H 8.22, N 11.82. Found: $\mathrm{C}$ 70.81, H 8.30, N 11.85.

ethyl 1-[(E)-1-(ethoxycarbonyl)-3-(naphthalen-1-yl)prop-2-en-2-yl]-1H-1,2,3triazole-4-carboxylate (4c)

oil. ${ }^{1} \mathrm{H}$ NMR (400 MHz, $\left.\mathrm{CDCl}_{3}\right): 8.58(\mathrm{~s}, 1 \mathrm{H}), 7.92-7.87(\mathrm{~m}, 3 \mathrm{H}), 7.65(\mathrm{~s}, 1 \mathrm{H})$, 7.54-7.48 (m, 4H), 4.44 (q, $J=7.2 \mathrm{~Hz}, 1 \mathrm{H}), 4.11$ (q, $J=7.2 \mathrm{~Hz}, 2 \mathrm{H}), 3.87(\mathrm{~s}, 2 \mathrm{H})$, $1.42(\mathrm{t}, J=7.2 \mathrm{~Hz}, 3 \mathrm{H}), 1.78(\mathrm{t}, J=7.2 \mathrm{~Hz}, 3 \mathrm{H}) .{ }^{13} \mathrm{C} \mathrm{NMR}\left(100 \mathrm{MHz}, \mathrm{CDCl}_{3}\right): 169.0$, $160.5,140.1,133.4,132.2,131.6,130.2,129.3,128.6,126.8,126.5,126.4,125.9$, 125.3, 124.3, 124.0, 61.5, 61.4, 35.7, 14.2, 13.9. IR (neat): 3141, 2982, 1736, 1591 $\mathrm{cm}^{-1}$. MS: m/z (\%) $379\left(\mathrm{M}^{+}, 9\right), 351$ (12), 165 (100). Anal. Calcd for $\mathrm{C}_{21} \mathrm{H}_{21} \mathrm{~N}_{3} \mathrm{O}_{4}$ (379.41): C 66.48, H 5.58, N 11.08. Found: C 66.41, H 5.53, N 11.15.

\section{ethyl 2,5-dimethyl-1H-pyrrole-3-carboxylate (5a) $)^{2,3}$}

m.p. $113-115^{\circ} \mathrm{C} .{ }^{1} \mathrm{H}$ NMR (400 MHz, $\mathrm{CDCl}_{3}$ ): 8.09 (br, 1H), 6.20 (m, 1H), 4.25 (q, J =7.2 Hz, 2H), 2.48 (s, 3H), 2.19 (s, 3H), 1.33 (t, $J=7.2 \mathrm{~Hz}, 3 \mathrm{H}) .{ }^{13} \mathrm{C}$ NMR (100 MHz, $\mathrm{CDCl}_{3}$ ): 165.9, $134.2,125.6,111.5,107.4,59.2,14.5,13.1,12.6$. 
ethyl 2-ethyl-5-methyl-1H-pyrrole-3-carboxylate (5b) ${ }^{4}$

m.p. 54-56 ${ }^{\circ} \mathrm{C} .{ }^{1} \mathrm{H}$ NMR (400 MHz, $\left.\mathrm{CDCl}_{3}\right): 8.05$ (br, $\left.1 \mathrm{H}\right), 6.20(\mathrm{~m}, 1 \mathrm{H}), 4.25$ (q, $J$ $=7.2 \mathrm{~Hz}, 2 \mathrm{H}), 2.94(\mathrm{q}, J=7.6 \mathrm{~Hz}, 2 \mathrm{H}), 2.21(\mathrm{~s}, 3 \mathrm{H}), 1.32(\mathrm{t}, J=7.2 \mathrm{~Hz}, 3 \mathrm{H}), 1.23(\mathrm{t}, J$ $=7.6 \mathrm{~Hz}, 3 \mathrm{H}) .{ }^{13} \mathrm{C} \mathrm{NMR}\left(100 \mathrm{MHz}, \mathrm{CDCl}_{3}\right): 165.6,140.1,125.5,110.8,107.5,59.2$, 20.5, 14.5, 13.6, 12.6. IR (neat): $3320,1671,1463,1223,1096 \mathrm{~cm}^{-1}$.

ethyl 5-ethyl-2-methyl-1H-pyrrole-3-carboxylate (5c)

Low melting point solid. ${ }^{1} \mathrm{H}$ NMR (400 MHz, $\left.\mathrm{CDCl}_{3}\right): 8.09$ (br, 1H), $6.23(\mathrm{~m}, 1 \mathrm{H})$, $4.25(\mathrm{q}, J=7.2 \mathrm{~Hz}, 2 \mathrm{H}), 2.54(\mathrm{q}, J=7.6 \mathrm{~Hz}, 2 \mathrm{H}), 2.49$ (s, 3H), 1.33 (t, $J=7.2 \mathrm{~Hz}, 3 \mathrm{H})$, $1.22(\mathrm{t}, J=7.6 \mathrm{~Hz}, 3 \mathrm{H}) .{ }^{13} \mathrm{C} \mathrm{NMR}\left(100 \mathrm{MHz}, \mathrm{CDCl}_{3}\right): 165.8,134.1,132.1,111.4$, 105.8, 59.2, 20.4, 14.5, 13.3, 13.1. IR (neat): $3294,1664,1459,1245,1096 \mathrm{~cm}^{-1} . \mathrm{MS}$ : m/z (\%) $181\left(\mathrm{M}^{+}, 83\right), 152$ (100). Anal. Calcd for $\mathrm{C}_{10} \mathrm{H}_{15} \mathrm{NO}_{2}$ (181.23): C 66.27, $\mathrm{H}$ 8.34, N 7.73. Found: C 66.16, H 8.42, 7.83.

ethyl 5-methyl-2-propyl-1H-pyrrole-3-carboxylate (5d) $)^{3,4}$

m.p. $72-74{ }^{\circ} \mathrm{C} .{ }^{1} \mathrm{H}$ NMR $\left(400 \mathrm{MHz}, \mathrm{CDCl}_{3}\right): 8.12(\mathrm{br}, 1 \mathrm{H}), 6.20(\mathrm{~m}, 1 \mathrm{H}), 4.24$ (q, $\left.J=7.2 \mathrm{~Hz}, 2 \mathrm{H}\right)$, $2.86(\mathrm{t}, J=7.6 \mathrm{~Hz}, 2 \mathrm{H}), 2.20(\mathrm{~s}, 3 \mathrm{H}), 1.68-1.59(\mathrm{~m}, 2 \mathrm{H}), 1.32(\mathrm{t}, J=7.2 \mathrm{~Hz}, 3 \mathrm{H}), 0.94(\mathrm{t}, J=7.6 \mathrm{~Hz}$, 3H). ${ }^{13} \mathrm{C}$ NMR (100 MHz, $\left.\mathrm{CDCl}_{3}\right): 165.7,138.9,125.5,111.1,107.5,59.1,29.2,22.9,14.4,13.9$, 12.6. 


\section{ethyl 5-methyl-2-octyl-1H-pyrrole-3-carboxylate (5e)}

Low melting point solid. ${ }^{1} \mathrm{H}$ NMR (400 MHz, $\left.\mathrm{CDCl}_{3}\right)$ : $7.99(\mathrm{br}, 1 \mathrm{H}), 6.20(\mathrm{~m}, 1 \mathrm{H})$, $4.24(\mathrm{q}, J=7.2 \mathrm{~Hz}, 2 \mathrm{H}), 2.88(\mathrm{t}, J=8.0 \mathrm{~Hz}, 2 \mathrm{H}), 2.20(\mathrm{~s}, 3 \mathrm{H}), 1.62-1.58(\mathrm{~m}, 2 \mathrm{H})$, 1.34-1.26 (m, 10H), $1.32(\mathrm{t}, J=7.2 \mathrm{~Hz}, 3 \mathrm{H}), 0.87(\mathrm{t}, J=6.8 \mathrm{~Hz}, 3 \mathrm{H}) .{ }^{13} \mathrm{C}$ NMR $(100$ $\left.\mathrm{MHz}, \mathrm{CDCl}_{3}\right): 165.6,139.1,125.5,111.1,107.5,59.1,31.8,29.6,29.5,29.4,29.2$, 27.3, 22.6, 14.5, 14.1, 12.6. IR (neat): $3315,1671,1462,1223,1084 \mathrm{~cm}^{-1} . \mathrm{MS}: \mathrm{m} / \mathrm{z}$ (\%) $265\left(\mathrm{M}^{+}, 29\right), 166(86), 69$ (100). Anal. Calcd for $\mathrm{C}_{16} \mathrm{H}_{27} \mathrm{NO}_{2}$ (265.39): C 72.41, H 10.25, N 5.28. Found: C 72.29, H 10.33, N 5.37.

\section{ethyl 5-isopropyl-2-methyl-1H-pyrrole-3-carboxylate (5f)}

Low melting point solid. ${ }^{1} \mathrm{H}$ NMR (400 MHz, $\left.\mathrm{CDCl}_{3}\right): 8.03(\mathrm{br}, 1 \mathrm{H}), 6.22(\mathrm{~m}, 1 \mathrm{H})$, $4.25(\mathrm{q}, J=7.2 \mathrm{~Hz}, 2 \mathrm{H}), 2.86-2.79(\mathrm{~m}, 1 \mathrm{H}), 2.49(\mathrm{~s}, 3 \mathrm{H}), 1.33(\mathrm{t}, J=7.2 \mathrm{~Hz}, 3 \mathrm{H}), 1.23$ (d, $J=7.2 \mathrm{~Hz}, 6 \mathrm{H}) .{ }^{13} \mathrm{C} \mathrm{NMR}\left(100 \mathrm{MHz}, \mathrm{CDCl}_{3}\right): 165.8,136.8,134.0,111.2,104.5$, 59.2, 26.7, 22.4, 14.5, 13.2. IR (neat): 3320, 1671, 1446, 1238, $1097 \mathrm{~cm}^{-1} . \mathrm{MS}: \mathrm{m} / \mathrm{z}$ (\%) $195\left(\mathrm{M}^{+}, 54\right), 180$ (100). Anal. Calcd for $\mathrm{C}_{11} \mathrm{H}_{17} \mathrm{NO}_{2}$ (195.26): C 67.66, H 8.78, N 7.17. Found: C 67.57, H 8.91, N 7.25.

\section{ethyl 4,5,6,7-tetrahydro-2-methyl-1H-indole-3-carboxylate $(5 \mathrm{~g})^{5}$}

m.p. $127-129{ }^{\circ} \mathrm{C} .{ }^{1} \mathrm{H}$ NMR $\left(400 \mathrm{MHz}, \mathrm{CDCl}_{3}\right): 8.25$ (br, $\left.1 \mathrm{H}\right), 4.25$ (q, J =7.2 Hz, 2H), 2.71-2.68 (m, 2H), $2.48(\mathrm{~m}, 5 \mathrm{H}), 1.76-1.74(\mathrm{~m}, 4 \mathrm{H}), 1.33(\mathrm{t}, J=7.2 \mathrm{~Hz}, 3 \mathrm{H}) .{ }^{13} \mathrm{C}$ NMR $\left(100 \mathrm{MHz}, \mathrm{CDCl}_{3}\right)$ : $166.5,134.2,125.4,118.4,109.3,58.9,23.4,23.2,22.9,22.3,14.5,13.5$. 
(Z)-ethyl 4,7,8,9-tetrahydro-2-methyl-1H-cycloocta[b]pyrrole-3-carboxylate (5h)

m.p. $154-156{ }^{\circ} \mathrm{C} .{ }^{1} \mathrm{H}$ NMR (400 MHz, $\mathrm{CDCl}_{3}$ ): 7.60 (br, 1H), 5.99-5.92 (m, 1H), 5.47-5.54 (m, 1H), $4.26(\mathrm{q}, J=7.2 \mathrm{~Hz}, 2 \mathrm{H}), 3.57(\mathrm{~d}, J=6.4 \mathrm{~Hz}, 2 \mathrm{H}), 2.67(\mathrm{t}, J=6.4 \mathrm{~Hz}$, 2H), $2.44(\mathrm{~s}, 3 \mathrm{H}), 2.21-2.16(\mathrm{~m}, 2 \mathrm{H}), 1.57-1.51(\mathrm{~m}, 2 \mathrm{H}), 1.34(\mathrm{t}, J=7.2 \mathrm{~Hz}, 3 \mathrm{H}) .{ }^{13} \mathrm{C}$ NMR (100 MHz, $\left.\mathrm{CDCl}_{3}\right): 166.2,132.5,130.2,128.8,125.3,120.6,110.8,59.0,25.6$, 25.5, 25.1, 25.0, 14.5, 14.0. IR (KBr): 3278, 1657, 1478, 1443, $1165 \mathrm{~cm}^{-1} . \mathrm{MS}: \mathrm{m} / \mathrm{z}$ (\%) $233\left(\mathrm{M}^{+}, 57\right), 166$ (100). Anal. Calcd for $\mathrm{C}_{14} \mathrm{H}_{19} \mathrm{NO}_{2}$ (233.31): C 72.07, H 8.21, N 6.00. Found: C 71.95, H 8.29, 6.05.

\section{ethyl 5-butyl-2-methyl-1H-pyrrole-3-carboxylate (5k)}

Low melting point solid. ${ }^{1} \mathrm{H}$ NMR (400 MHz, $\left.\mathrm{CDCl}_{3}\right)$ : 7.97 (br, 1H), $6.22(\mathrm{~m}, 1 \mathrm{H})$, 4.25 (q, $J=7.2 \mathrm{~Hz}, 2 \mathrm{H}), 2.51(\mathrm{t}, J=7.2 \mathrm{~Hz}, 2 \mathrm{H}), 2.49(\mathrm{~s}, 3 \mathrm{H}), 1.61-1.53(\mathrm{~m}, 2 \mathrm{H})$, 1.39-1.32 (m, 2H), $1.33(\mathrm{t}, J=7.2 \mathrm{~Hz}, 3 \mathrm{H}), 0.92(\mathrm{t}, J=7.6 \mathrm{~Hz}, 3 \mathrm{H}) .{ }^{13} \mathrm{C}$ NMR (100 $\left.\mathrm{MHz}, \mathrm{CDCl}_{3}\right): 165.8,134.0,130.7,111.5,106.5,59.2,31.4,27.0,22.3,14.5,13.8$ 13.2. IR (neat): $3324,1669,1446,1232,1095 \mathrm{~cm}^{-1} . \mathrm{MS}: \mathrm{m} / \mathrm{z}(\%) 209\left(\mathrm{M}^{+}, 41\right), 166$ (100). Anal. Calcd for $\mathrm{C}_{12} \mathrm{H}_{19} \mathrm{NO}_{2}$ (209.28): C 68.87, H 9.15, N 6.69. Found: C 69.01, H 9.30, N 6.75 .

ethyl 5-hexyl-2-methyl-1H-pyrrole-3-carboxylate (5l) ${ }^{6}$ 
m.p. $54-56{ }^{\circ} \mathrm{C} .{ }^{1} \mathrm{H}$ NMR (400 MHz, $\left.\mathrm{CDCl}_{3}\right): 8.00$ (br, 1H), $6.21(\mathrm{~m}, 1 \mathrm{H}), 4.25$ (q, $J$ $=7.2 \mathrm{~Hz}, 2 \mathrm{H}), 2.50(\mathrm{t}, J=7.6 \mathrm{~Hz}, 2 \mathrm{H}), 2.48(\mathrm{~s}, 3 \mathrm{H}), 1.62-1.54(\mathrm{~m}, 2 \mathrm{H}), 1.34-1.28(\mathrm{~m}$, $6 \mathrm{H}), 1.33(\mathrm{t}, J=7.2 \mathrm{~Hz}, 3 \mathrm{H}), 0.88(\mathrm{t}, J=7.2 \mathrm{~Hz}, 3 \mathrm{H}) .{ }^{13} \mathrm{C} \mathrm{NMR}\left(100 \mathrm{MHz}, \mathrm{CDCl}_{3}\right)$ : $165.8,134.0,130.8,111.5,106.5,59.2,31.6,29.3,28.9,27.3,22.5,14.5,14.0,13.2$.

\section{ethyl 2-methyl-5-(6-methylhept-5-en-2-yl)-1H-pyrrole-3-carboxylate (5m)}

Low melting point solid. ${ }^{1} \mathrm{H}$ NMR (400 MHz, $\left.\mathrm{CDCl}_{3}\right)$ : 7.97 (br, 1H), $6.22(\mathrm{~m}, 1 \mathrm{H})$, 5.09-5.06 (m, 1H), 4.25 (q, J=7.2 Hz, 2H), 2.69-2.63 (m, 1H), 2.48 (s, 3H), 1.98-1.92 $(\mathrm{m}, 2 \mathrm{H}), 1.68(\mathrm{~s}, 3 \mathrm{H}), 1.56(\mathrm{~s}, 3 \mathrm{H}), 1.62-1.48(\mathrm{~m}, 2 \mathrm{H}), 1.33(\mathrm{t}, J=7.2 \mathrm{~Hz}, 3 \mathrm{H}), 1.21(\mathrm{~d}$, $J=6.8 \mathrm{~Hz}, 3 \mathrm{H}) .{ }^{13} \mathrm{C} \mathrm{NMR}\left(100 \mathrm{MHz}, \mathrm{CDCl}_{3}\right): 165.8,135.6,133.8,131.8,124.1$, $111.3,105.3,59.2,37.2,31.7,25.68,25.65,20.4,17.7,14.5,13.2$. IR (neat): 3316 , 1670, 1449, 1233, $1091 \mathrm{~cm}^{-1}$. MS: m/z (\%) $263\left(\mathrm{M}^{+}, 37\right), 180$ (97), 108 (100). Anal. Calcd for $\mathrm{C}_{16} \mathrm{H}_{25} \mathrm{NO}_{2}$ (263.38): C 72.96, H 9.57, N 5.32. Found: C 72.91, H 9.68, N 5.41.

\section{Reference:}

1. Lang, R. W.; Hansen, H.-J. Org. Synth. 1984, 62, 202.

2. Alberola A.; Ortega A. G., Sádaba M. L.; Sañudo C. Tetrahedron 1999, 55, 6555.

3. Yu, M.; Pagenkopf, B. L. Org. Lett. 2003, 5, 5099.

4. Roomi, M. W.; Macdonald, S. F. Can. J. of Chem. 1970, 48, 1689.

5. Chiu P.-K.; Sannes M. P. Tetrahedron 1990, 46, 3439.

6. Araki S.; Tanaka T.; Toumatsu S.; Hirashita T. Org. Biomol. Chem. 2003, 1, 4025. 
3. ${ }^{1} \mathrm{H}$ NMR and ${ }^{13} \mathrm{C}$ NMR spectra of key compounds
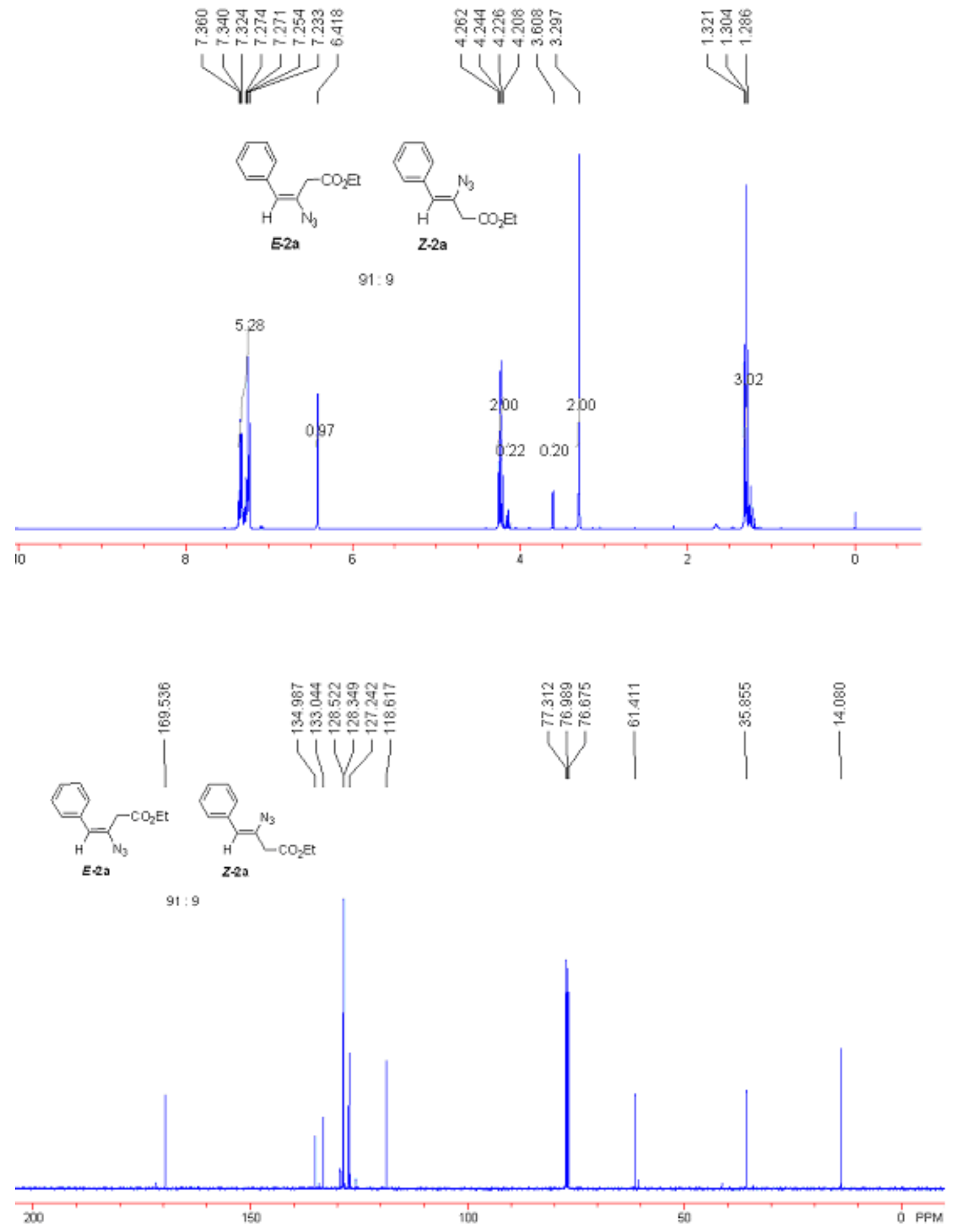


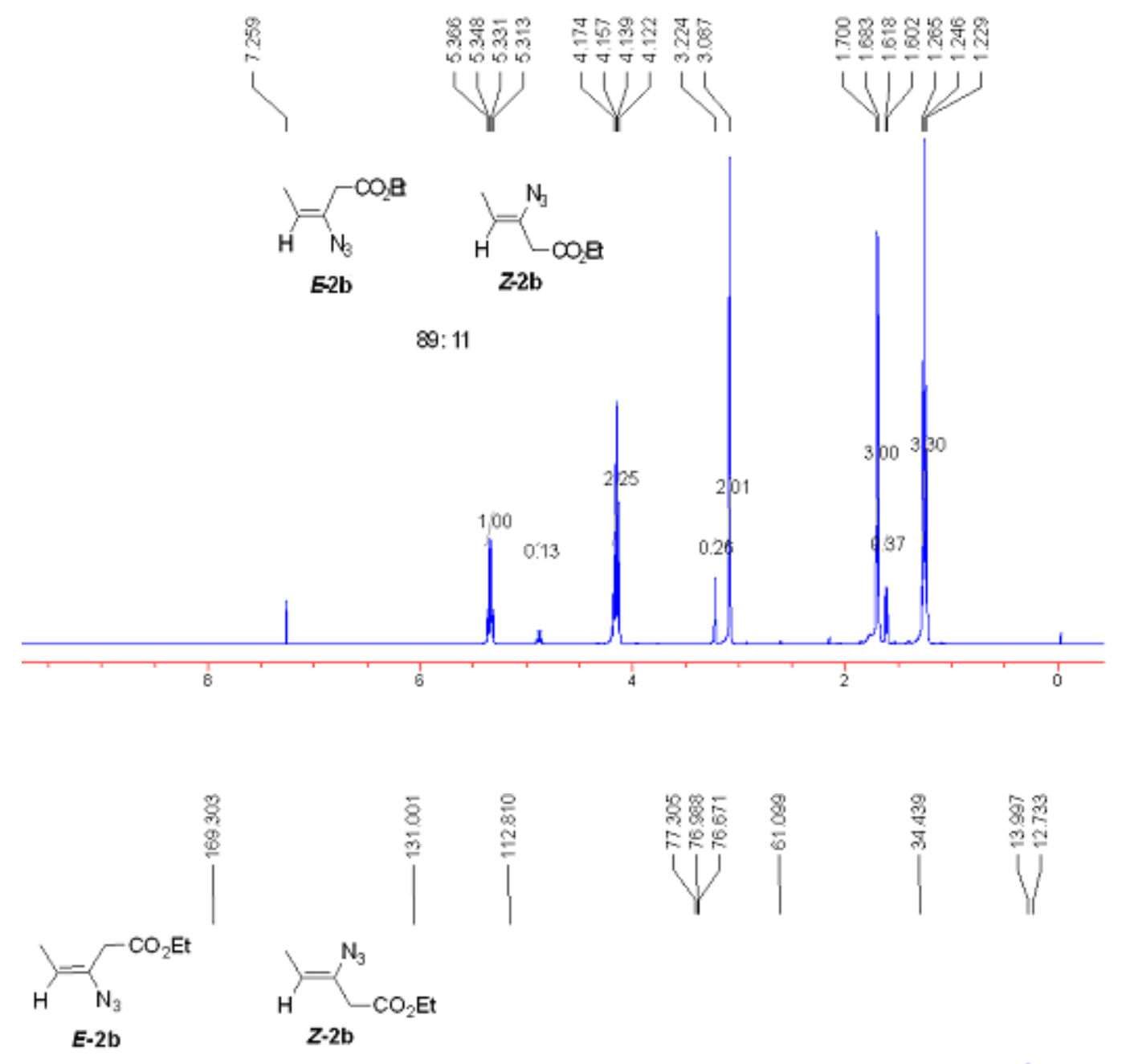

$89: 11$

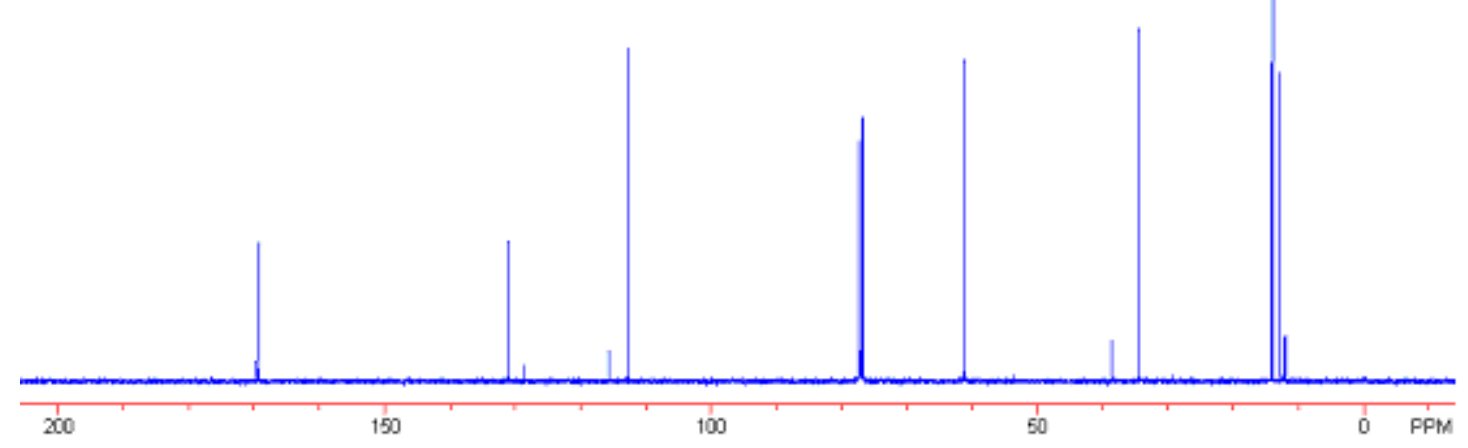




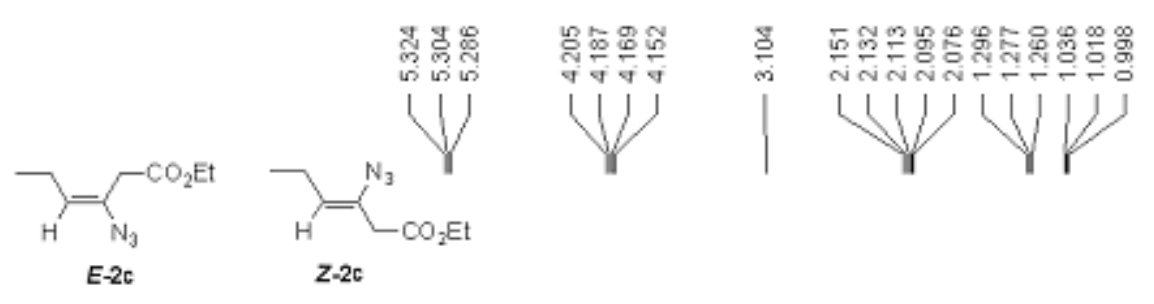

$93: 7$
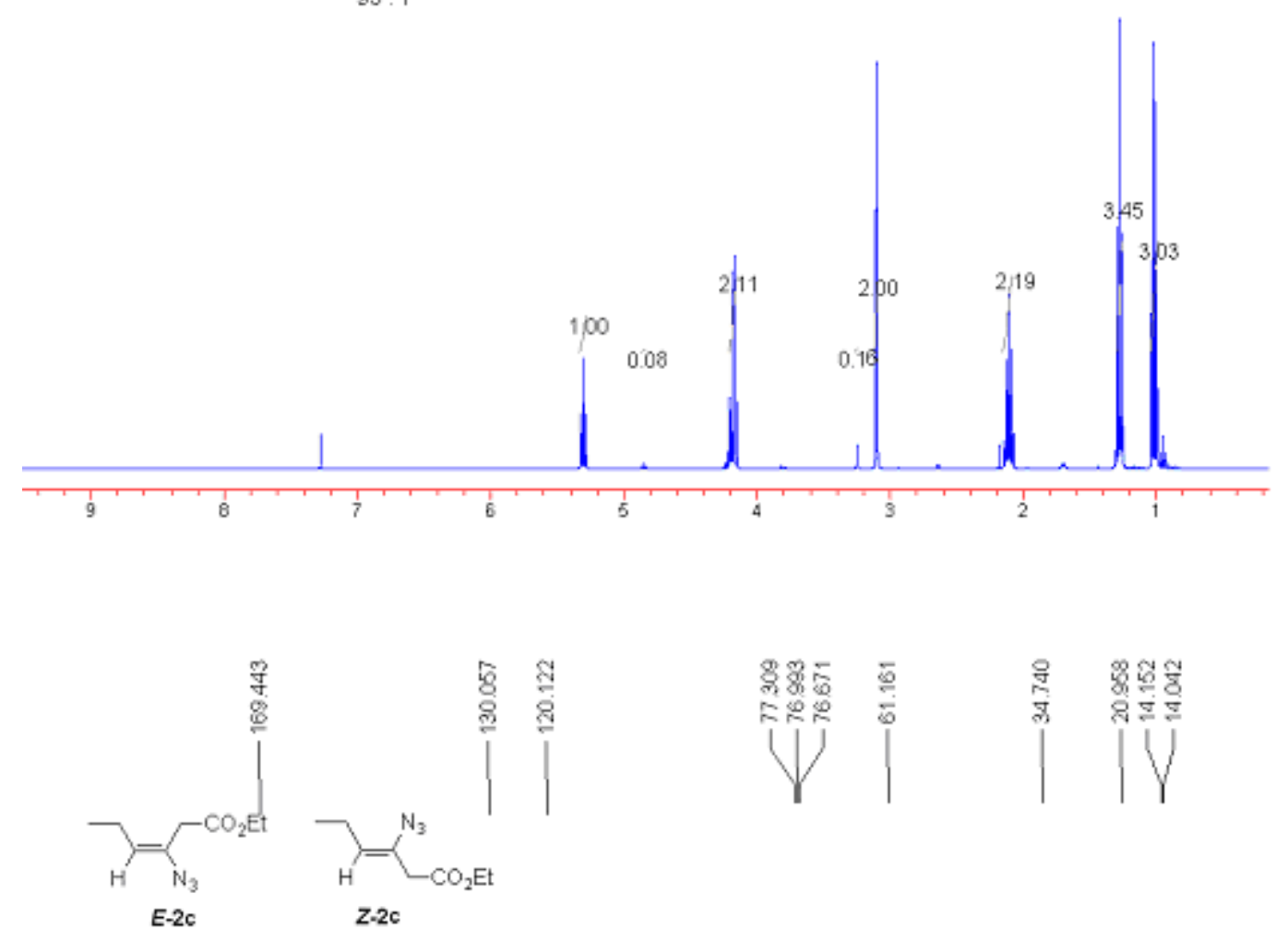

$93: 7$

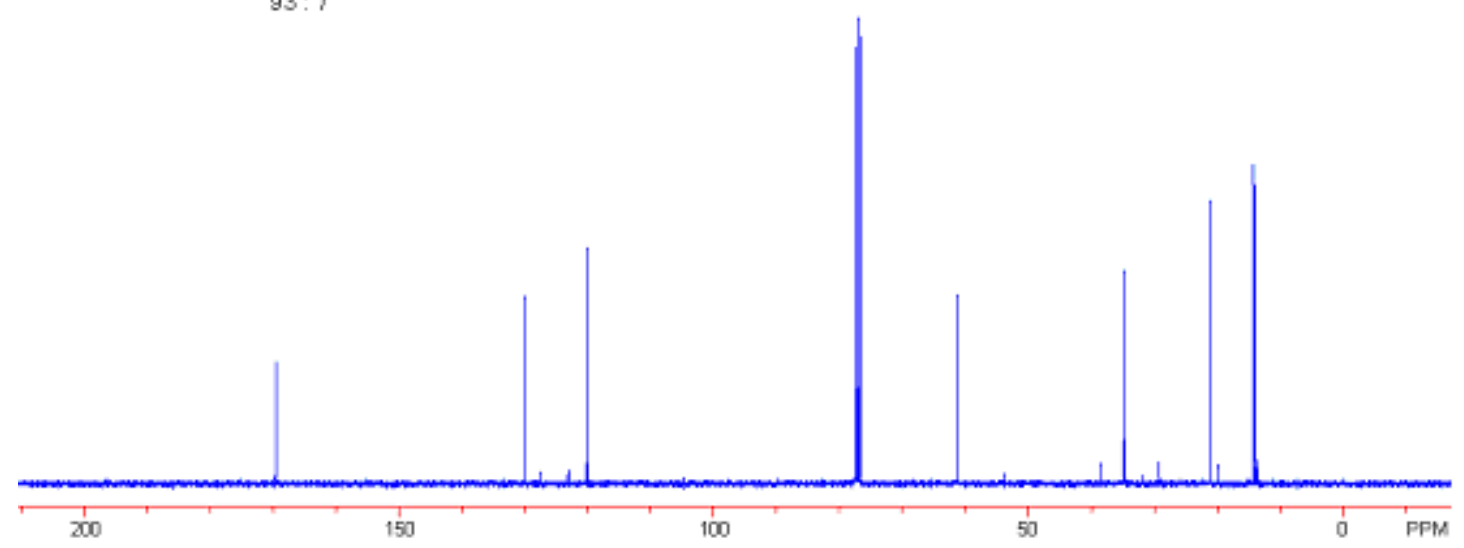




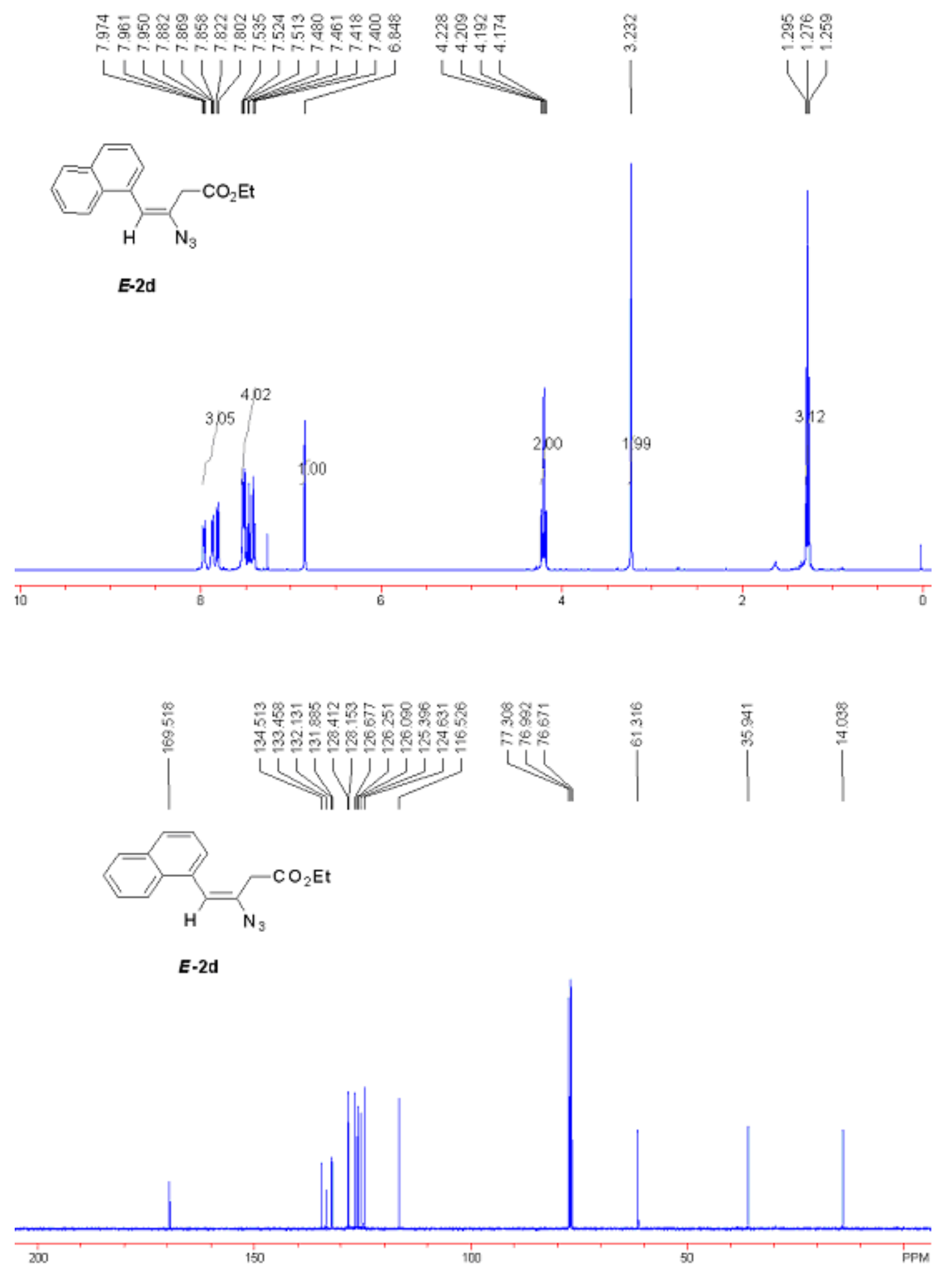




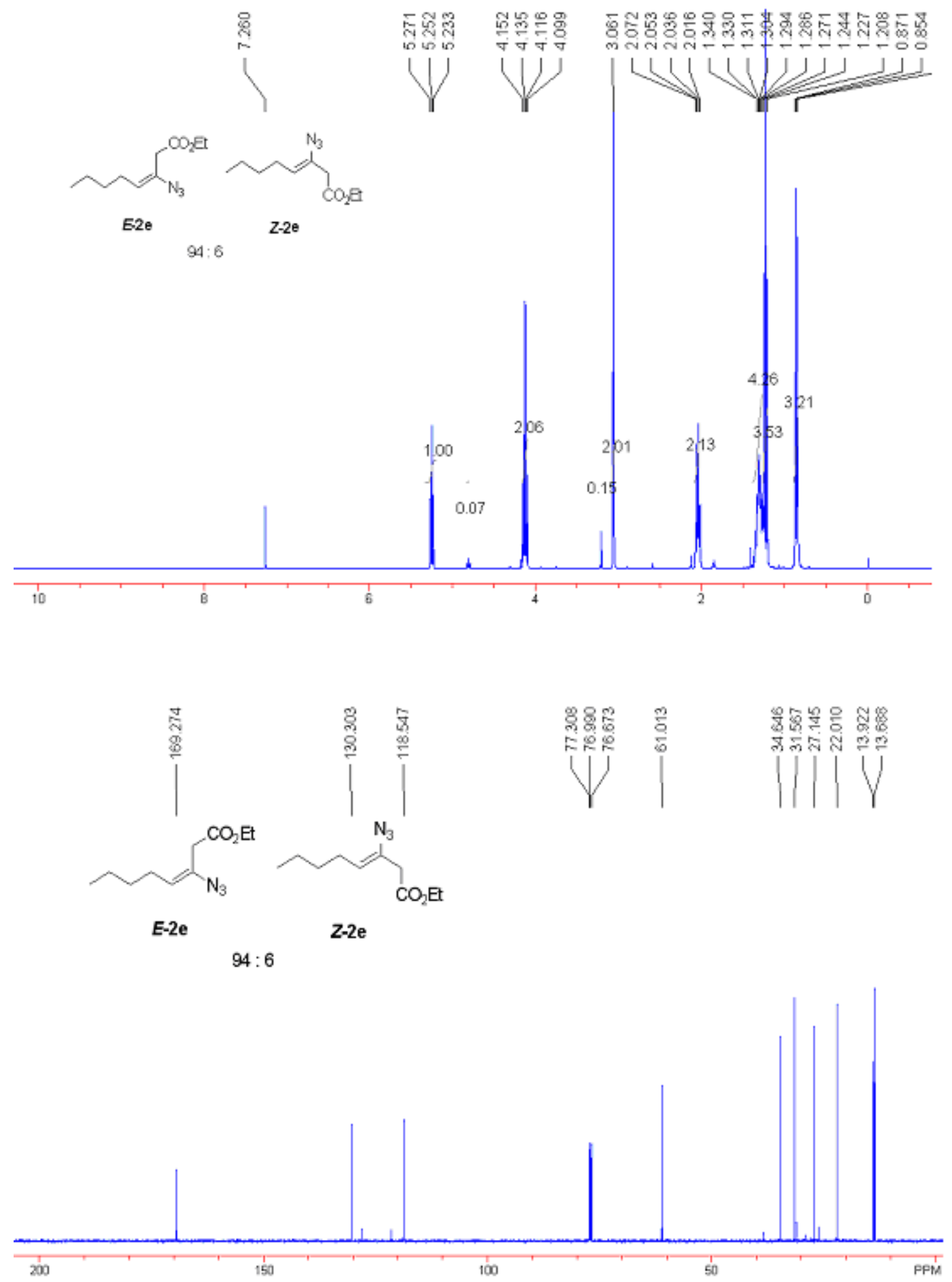



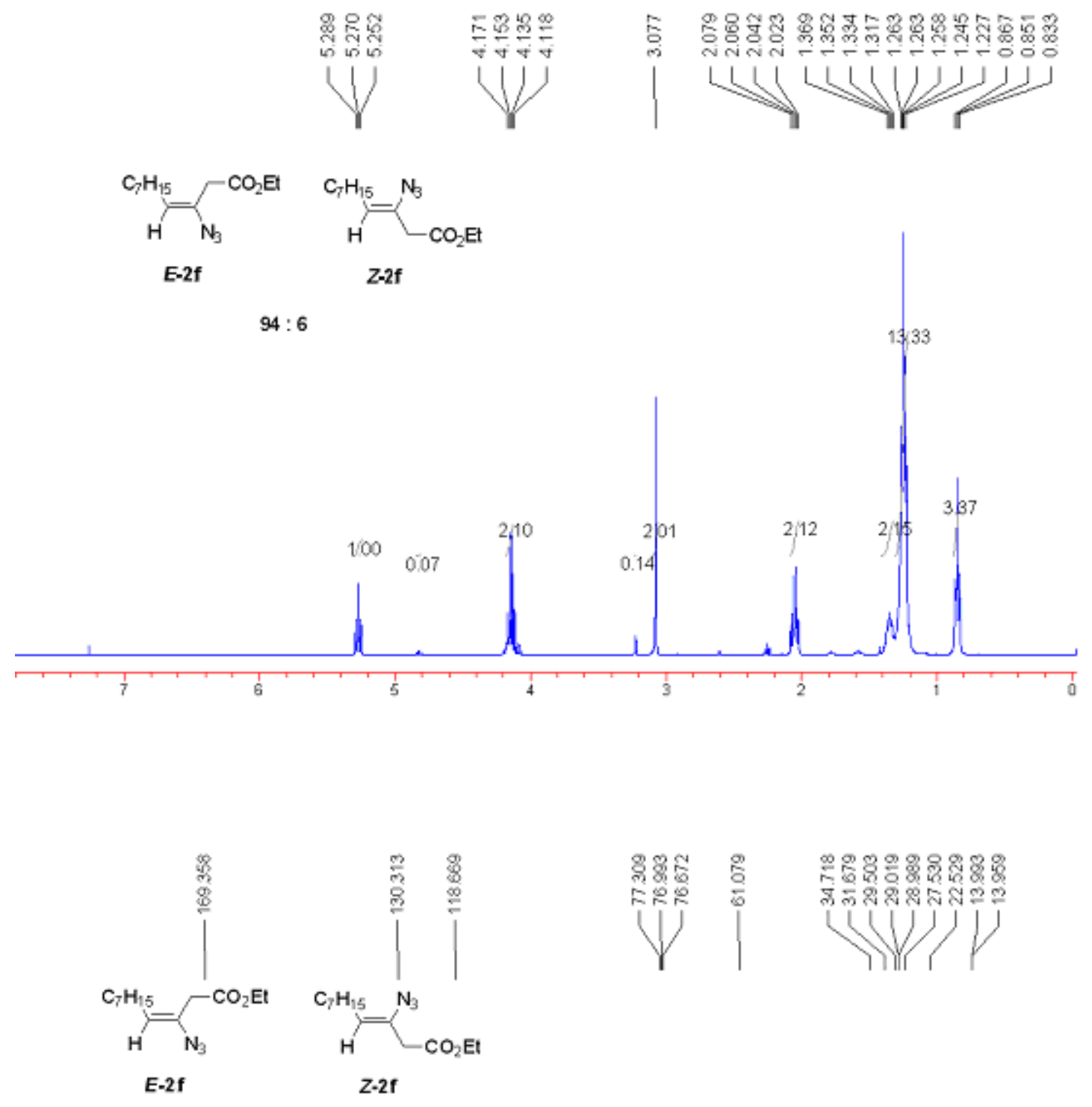

$94: 6$

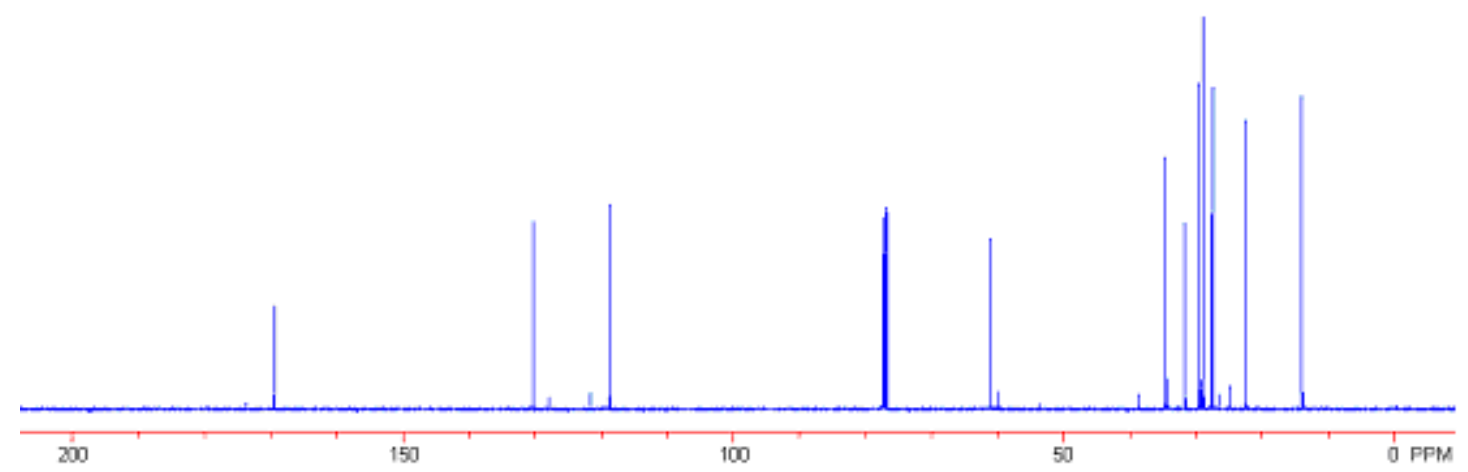



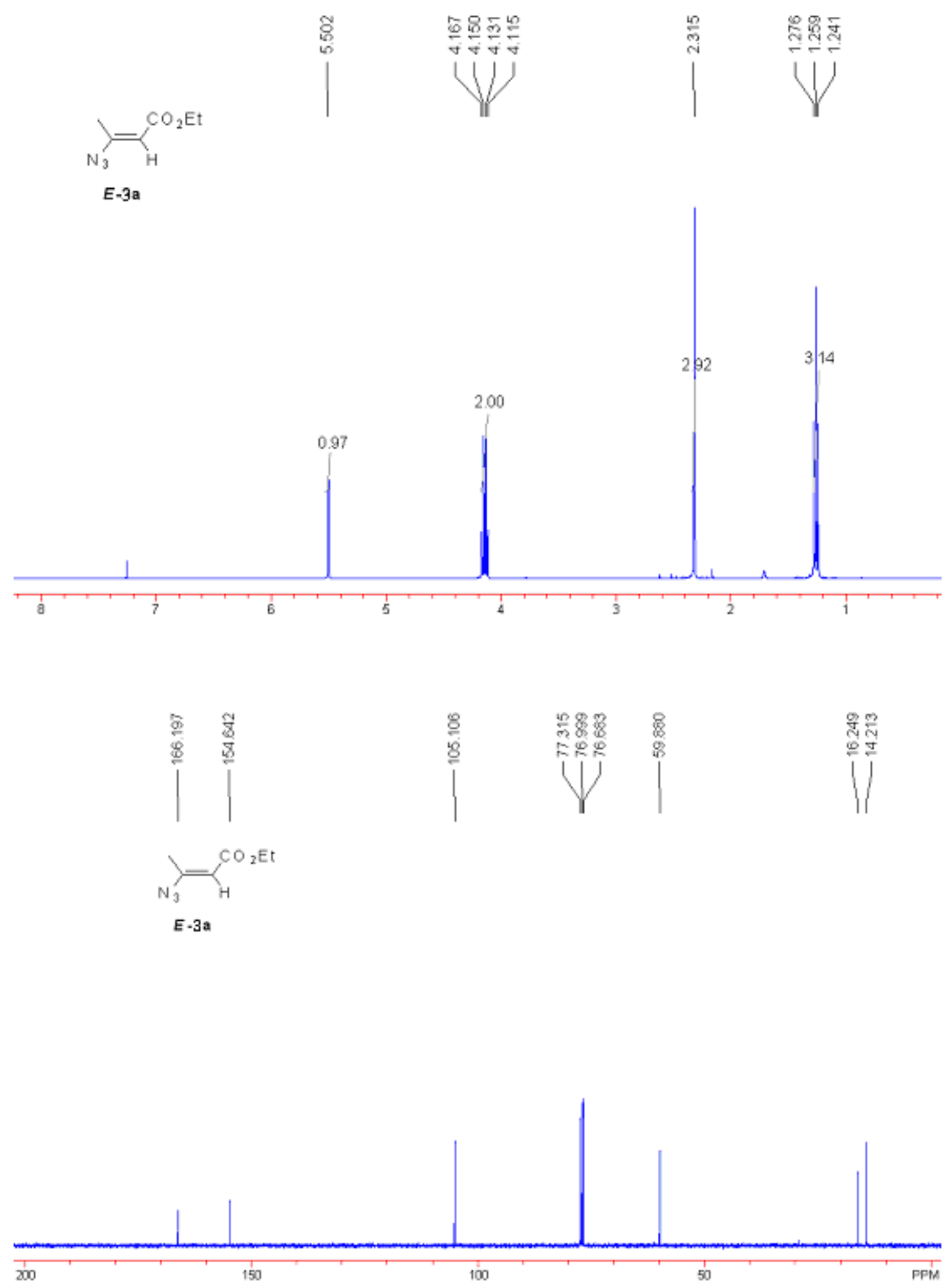


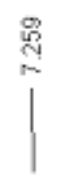

要果器举

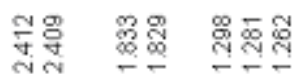

U

V V V

$\mathrm{N}_{3}=\mathrm{CO}^{\mathrm{CO}_{2} \mathrm{Et}}$

E-3b
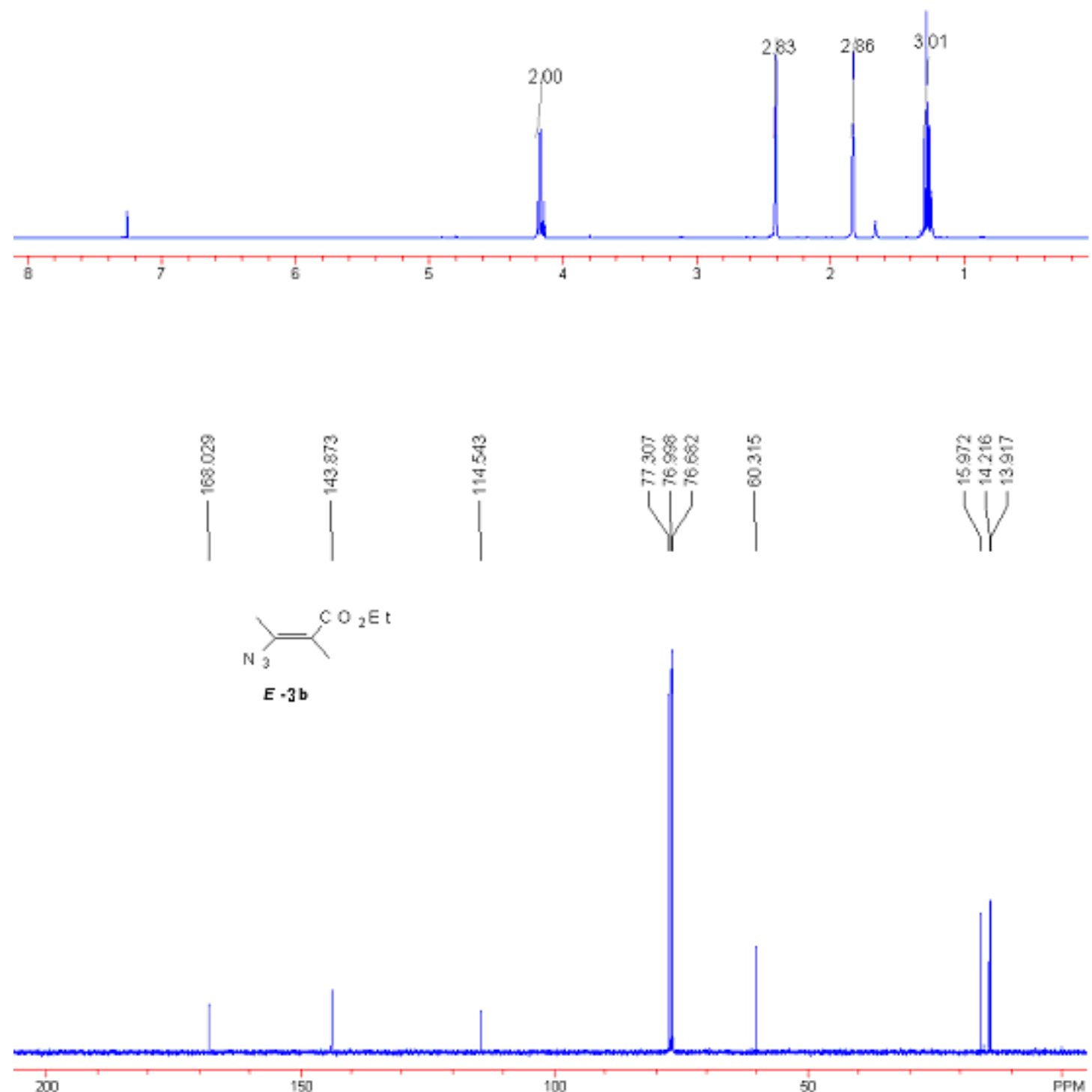

S21 

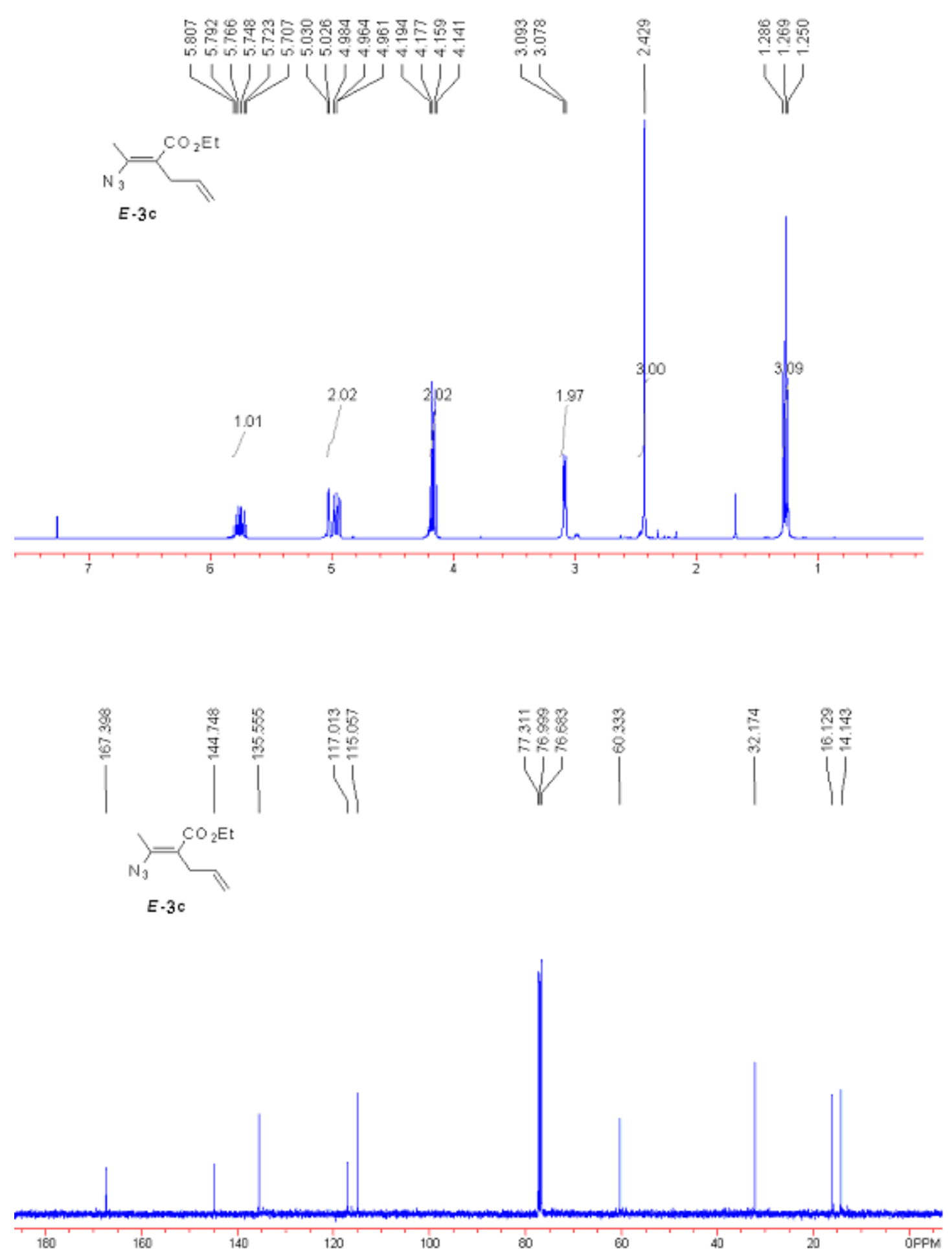


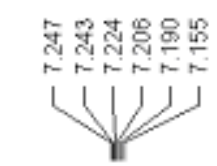

$\underbrace{7}$

충혼

\rangle$_{N_{3}}=\langle\underbrace{\mathrm{CO}_{2} \mathrm{Et}}_{E \cdot 3 \mathrm{~d}}$
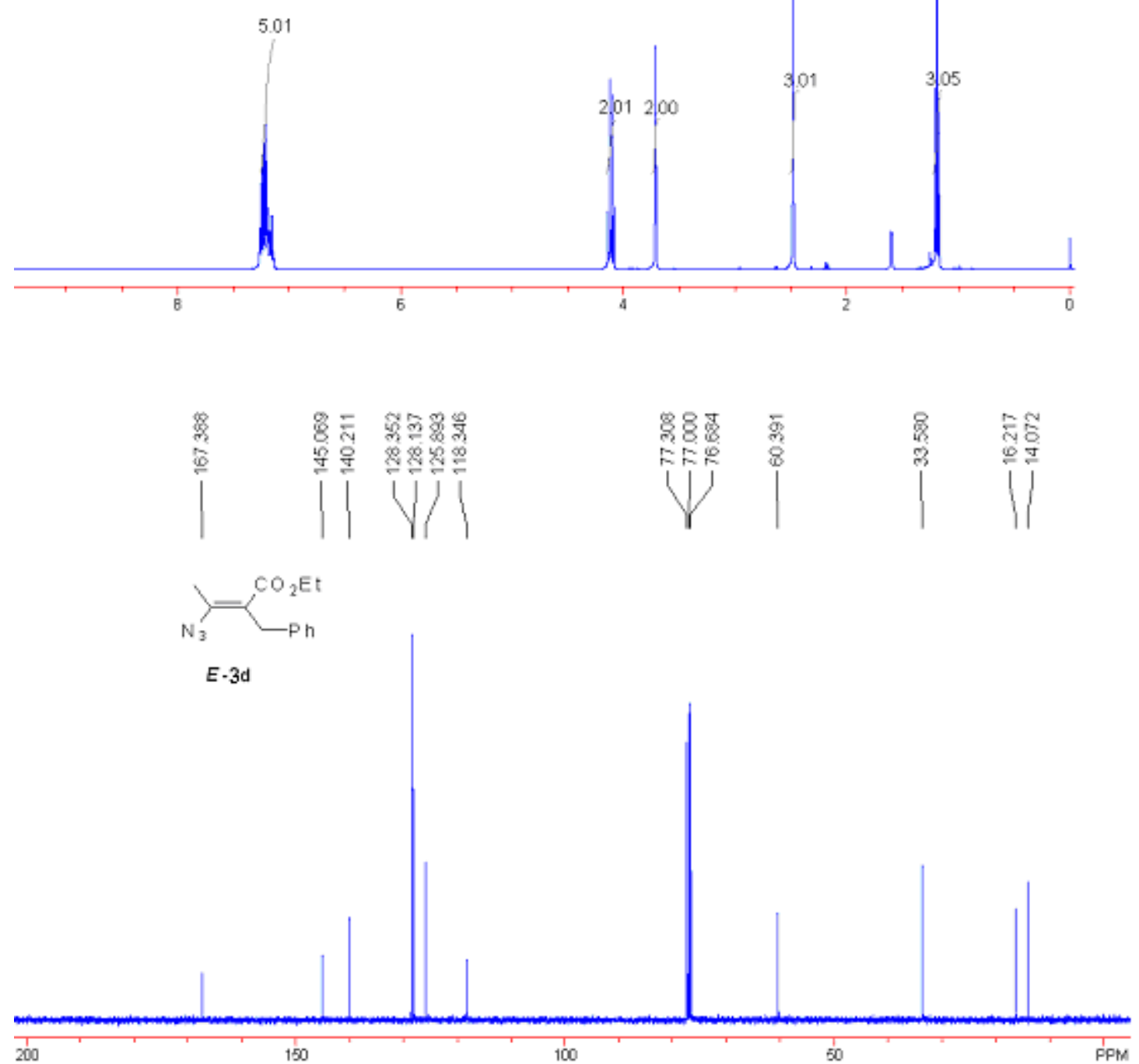


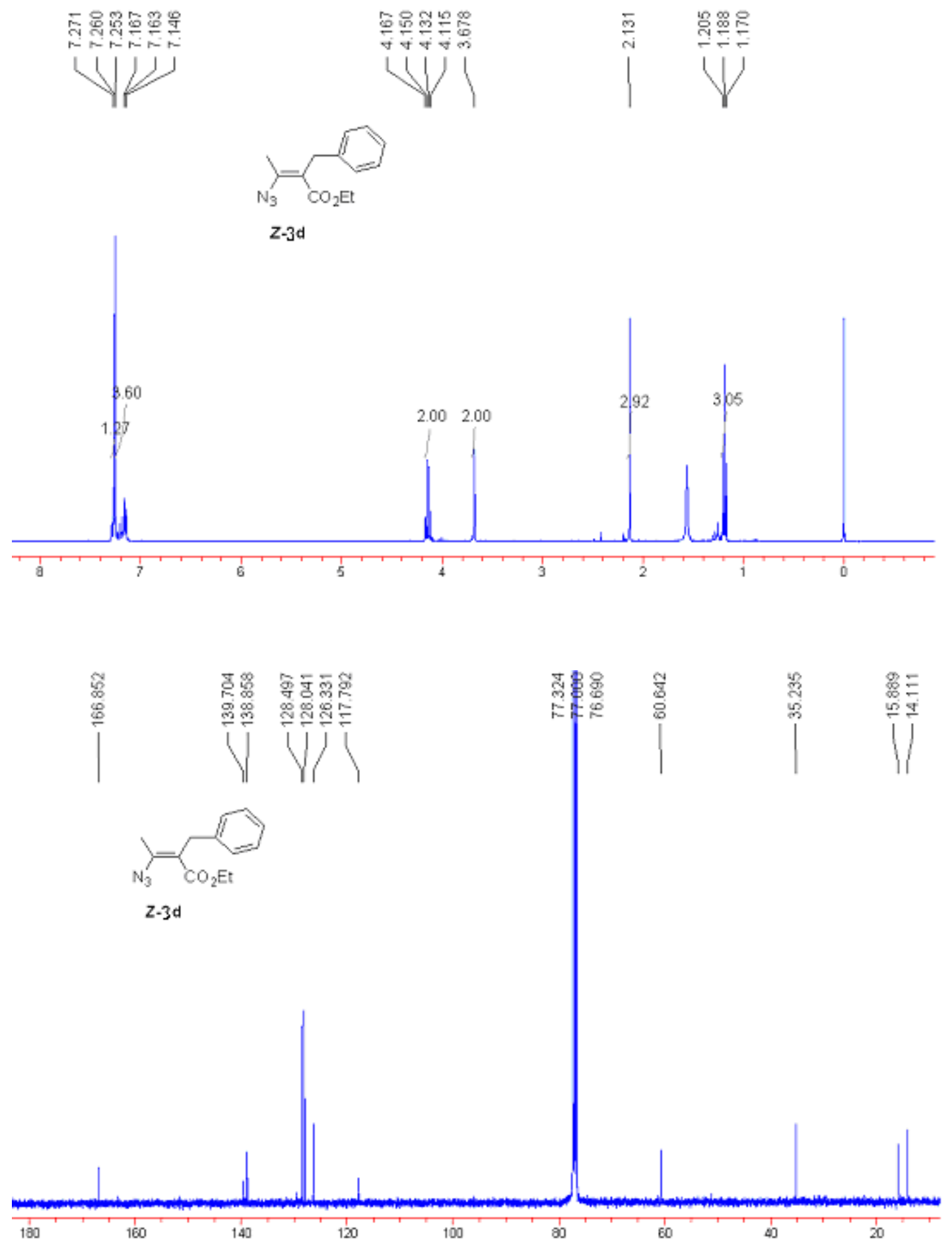




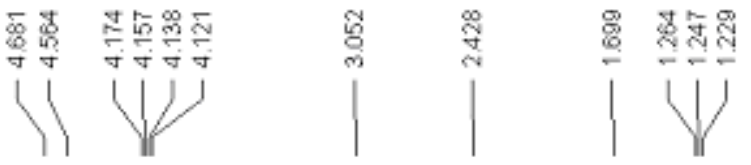
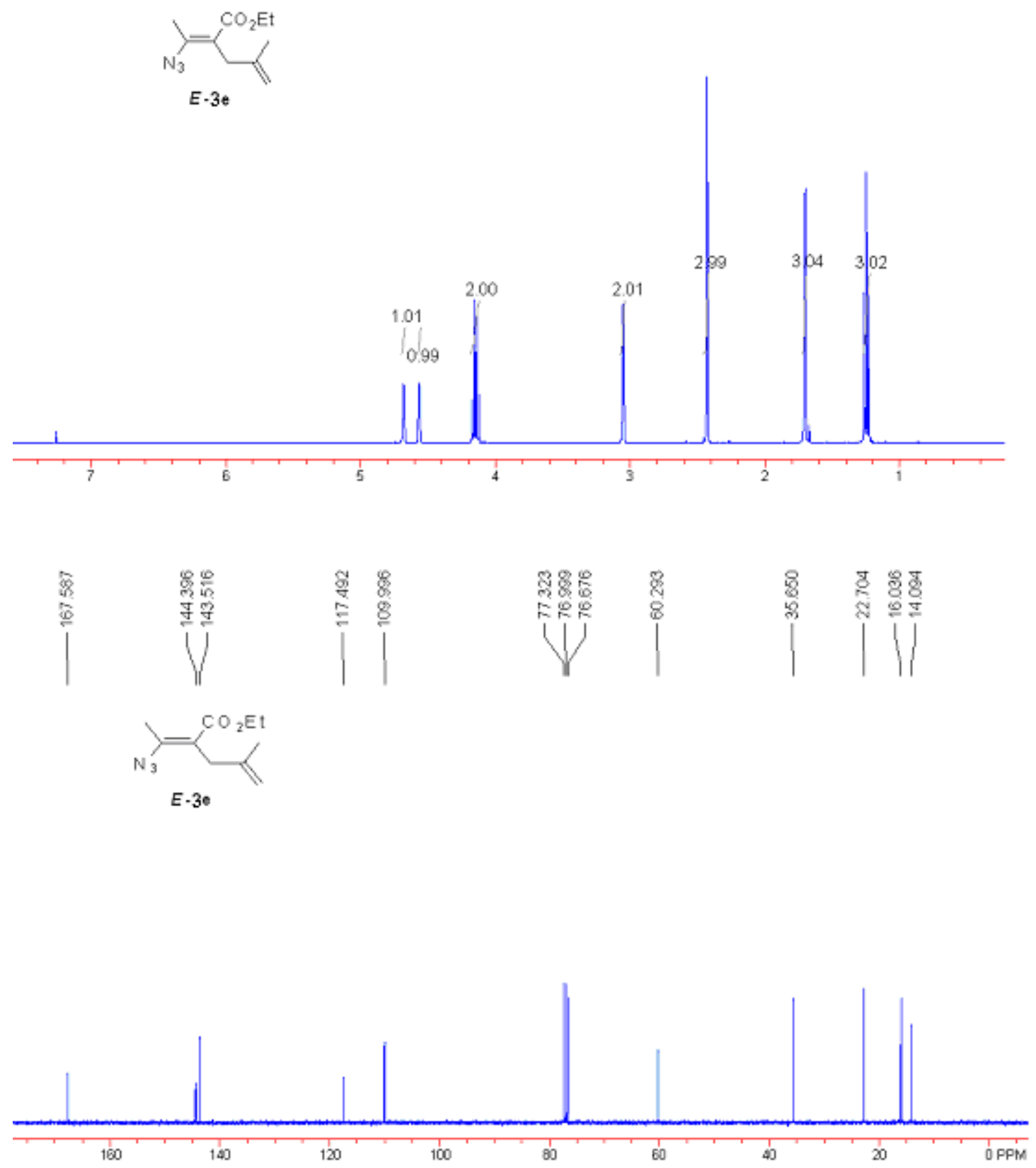


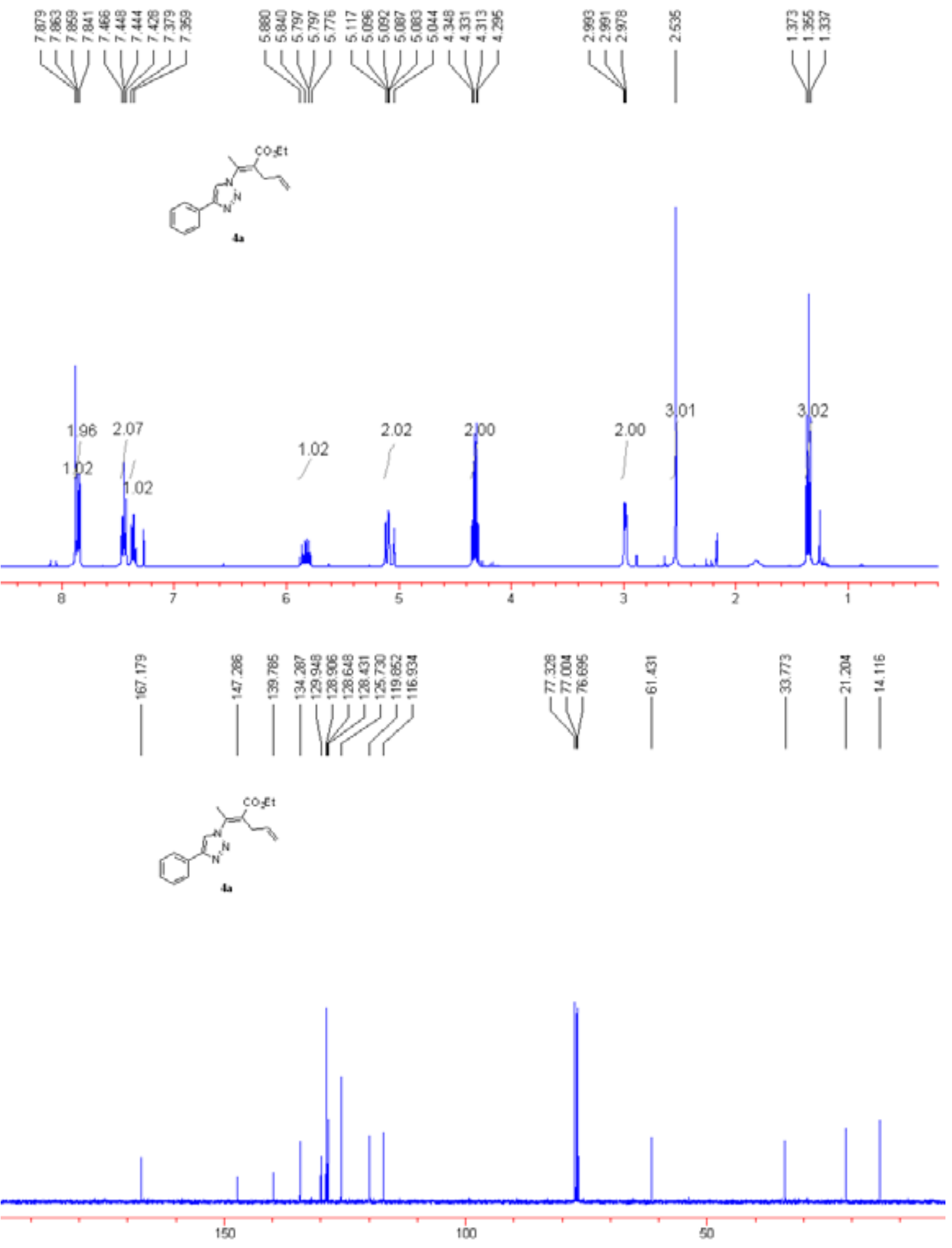



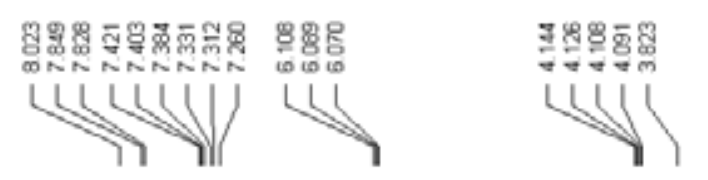

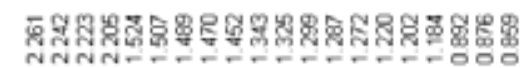

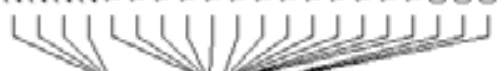
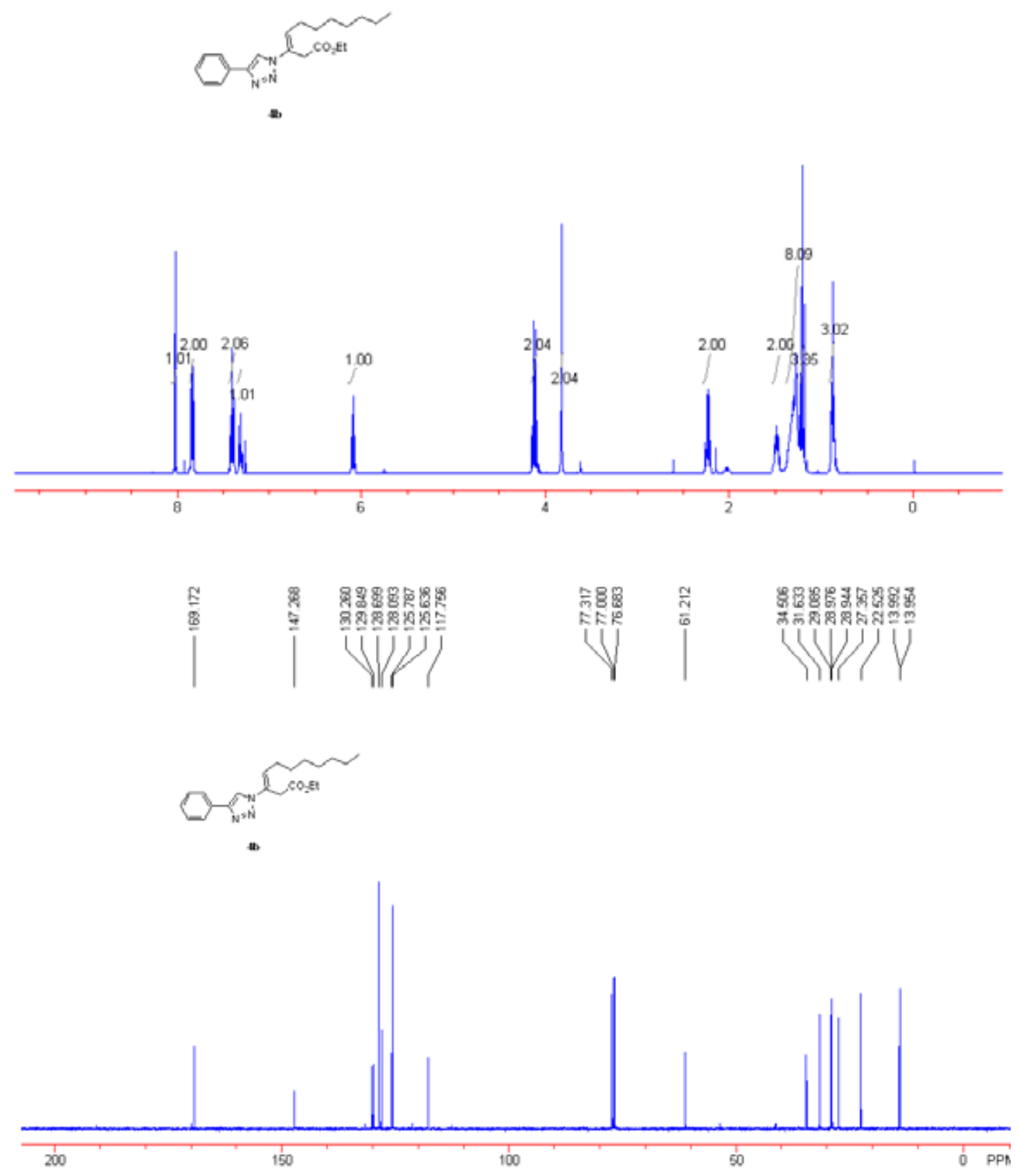

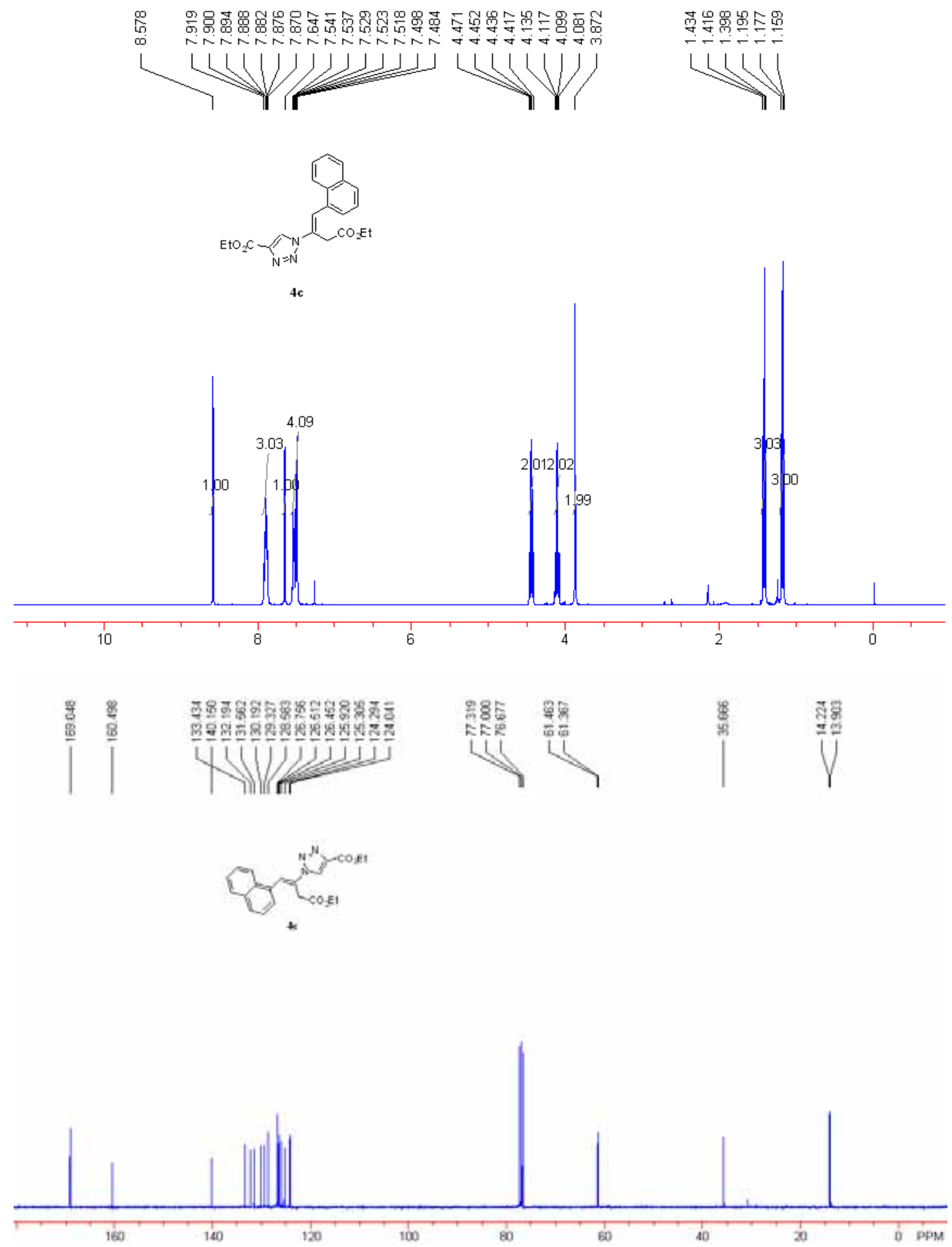

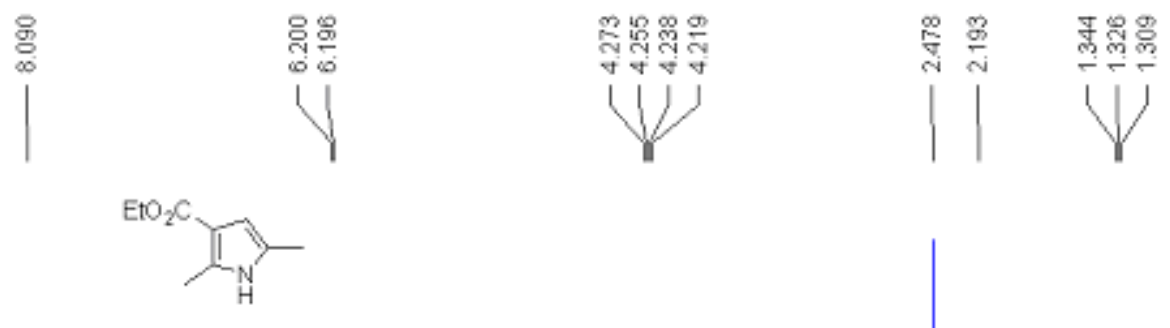

$5 a$
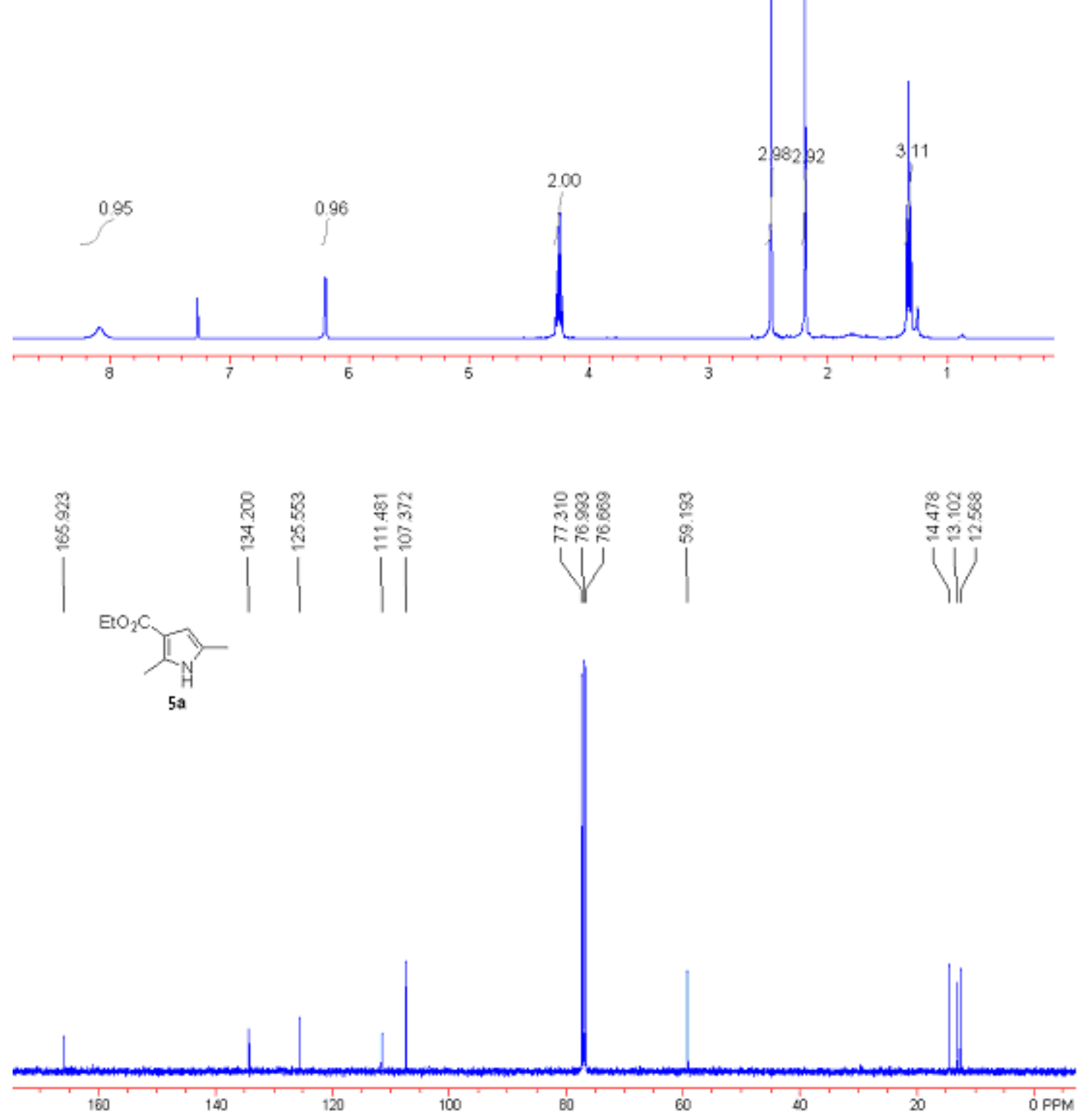


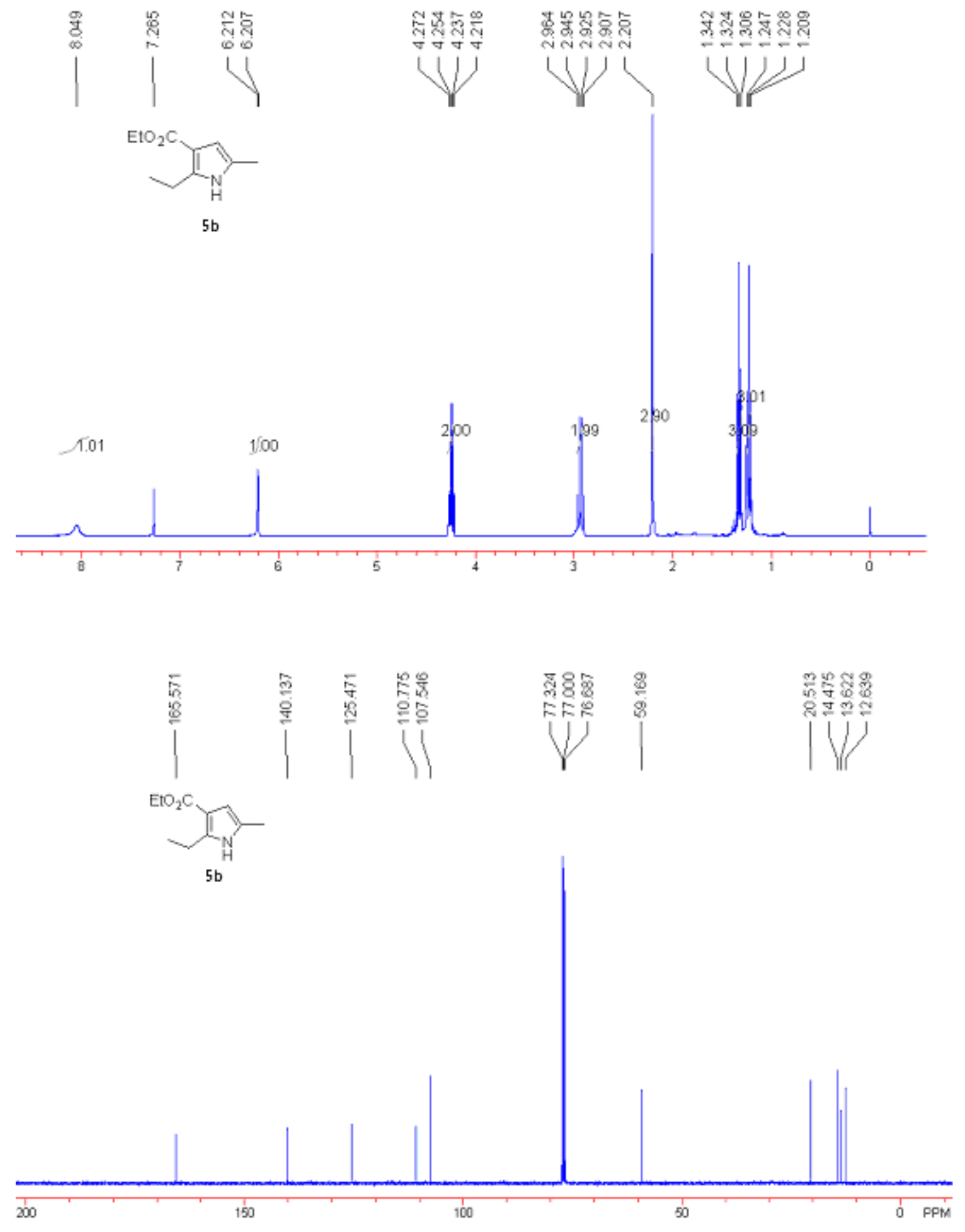




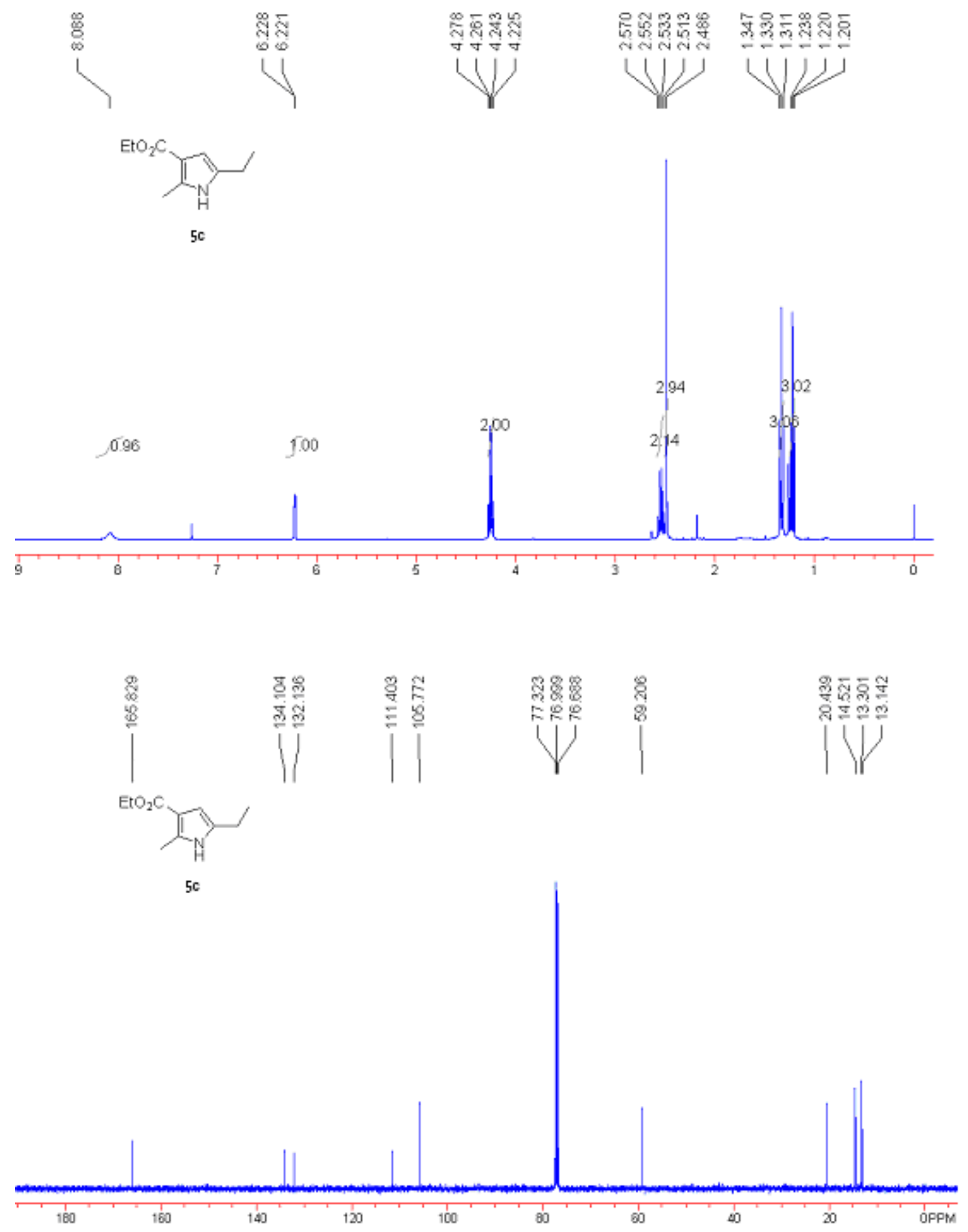




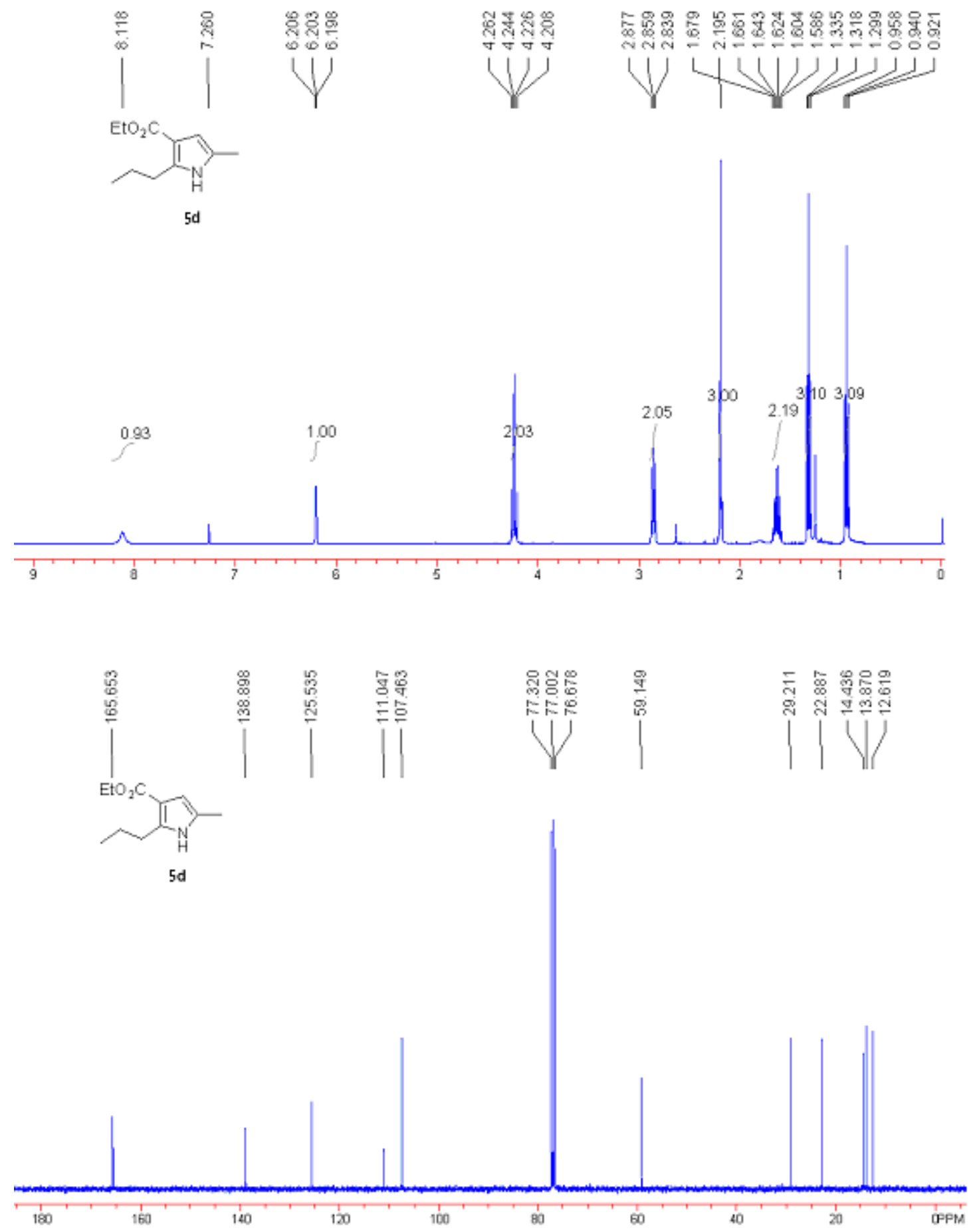




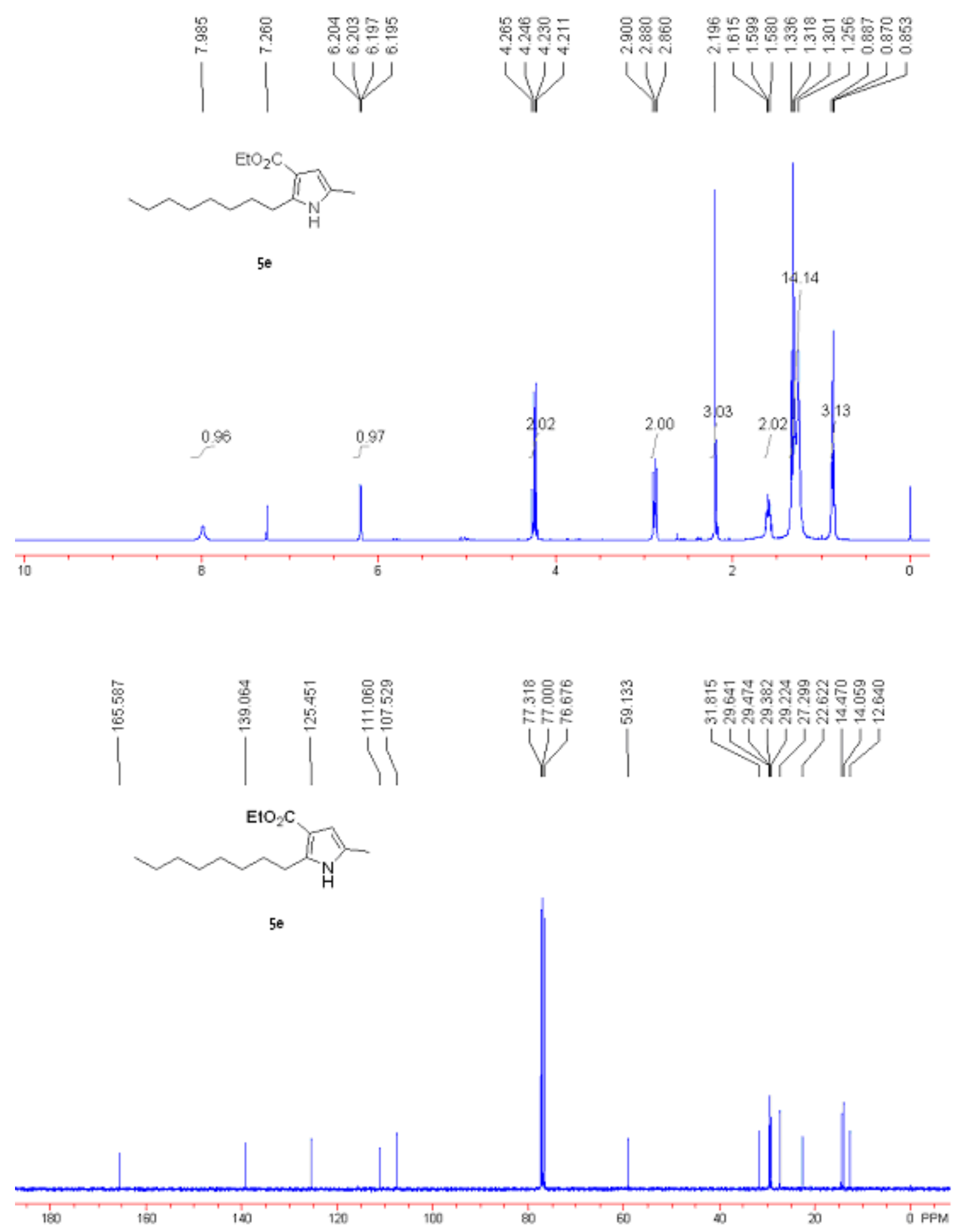




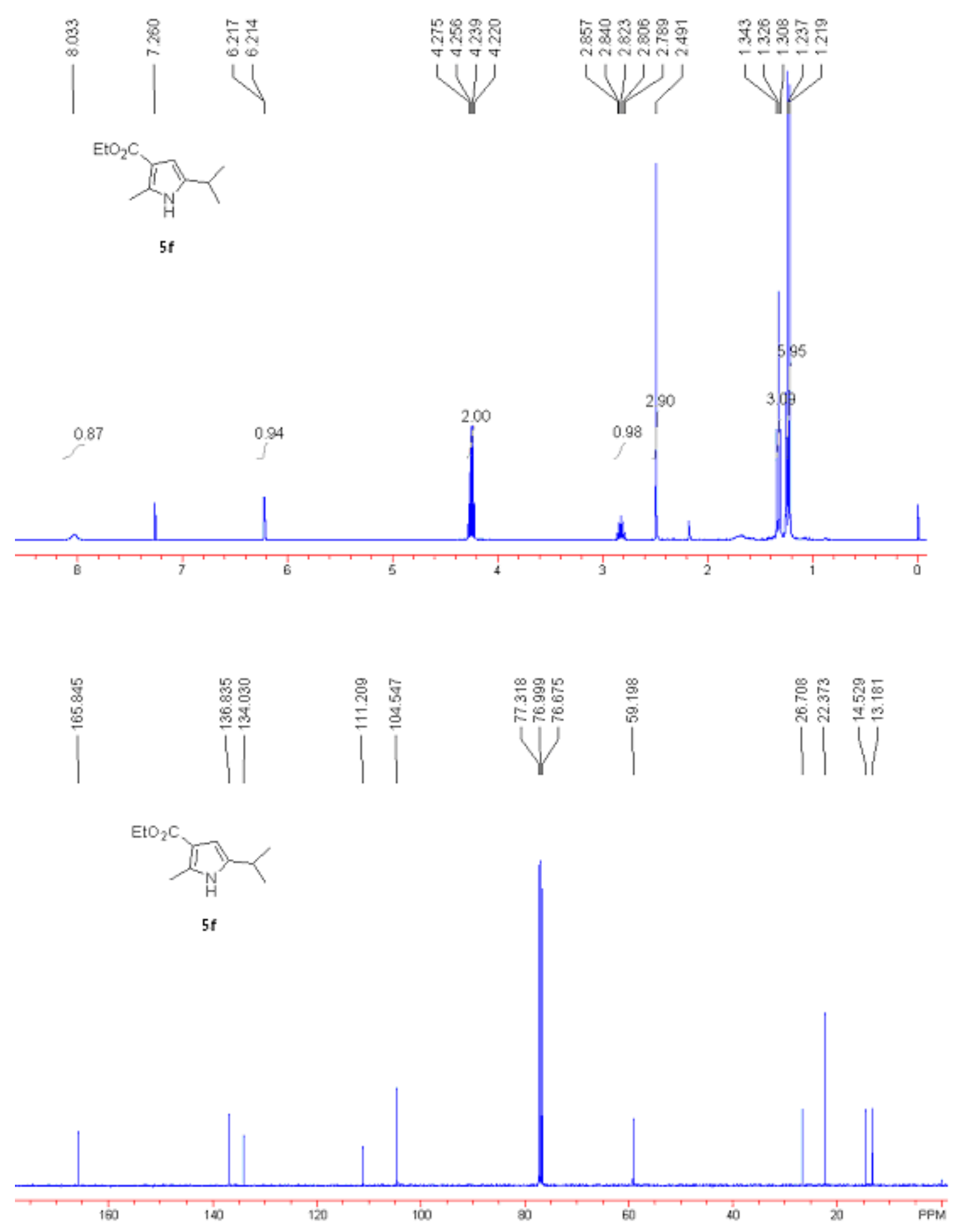




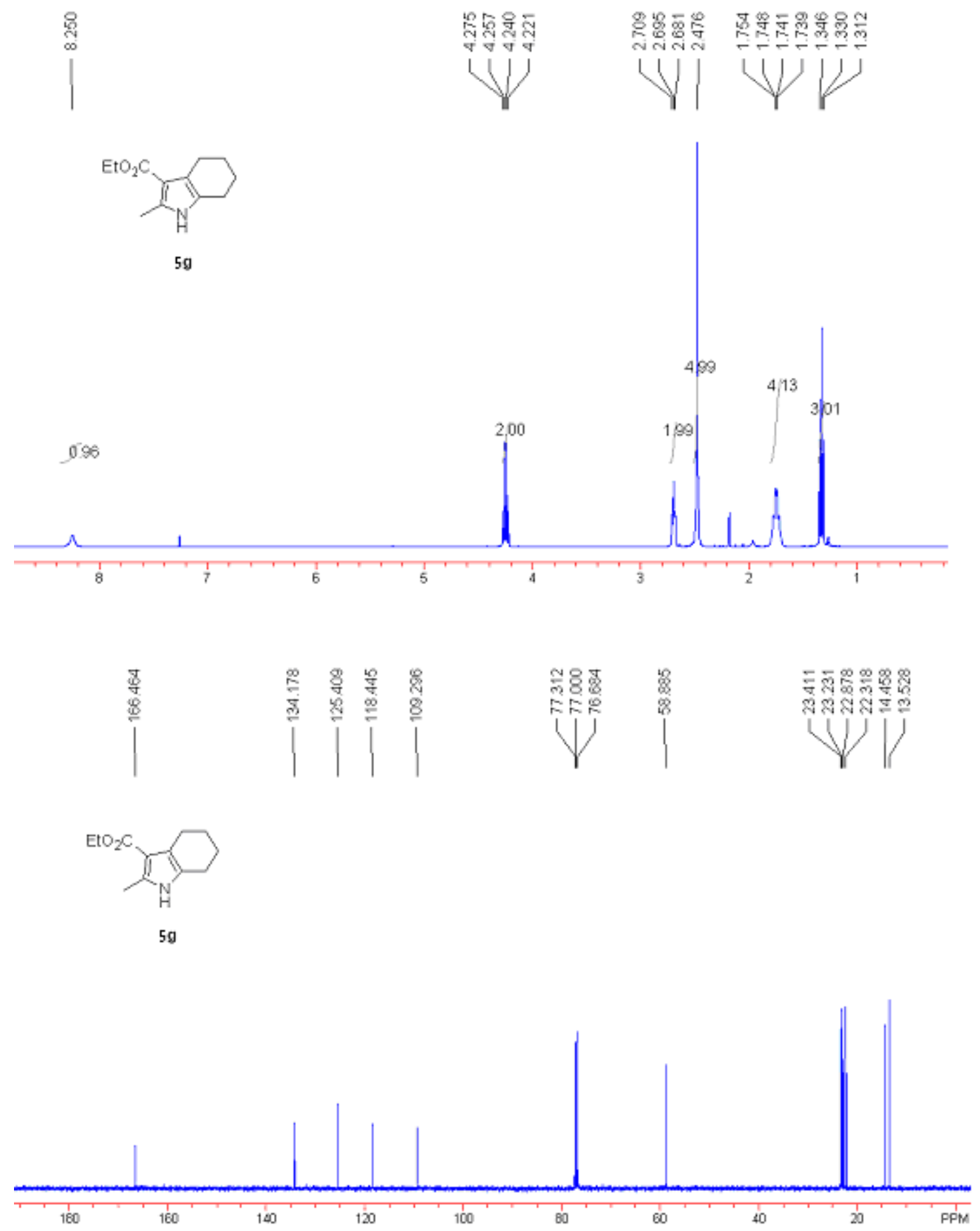




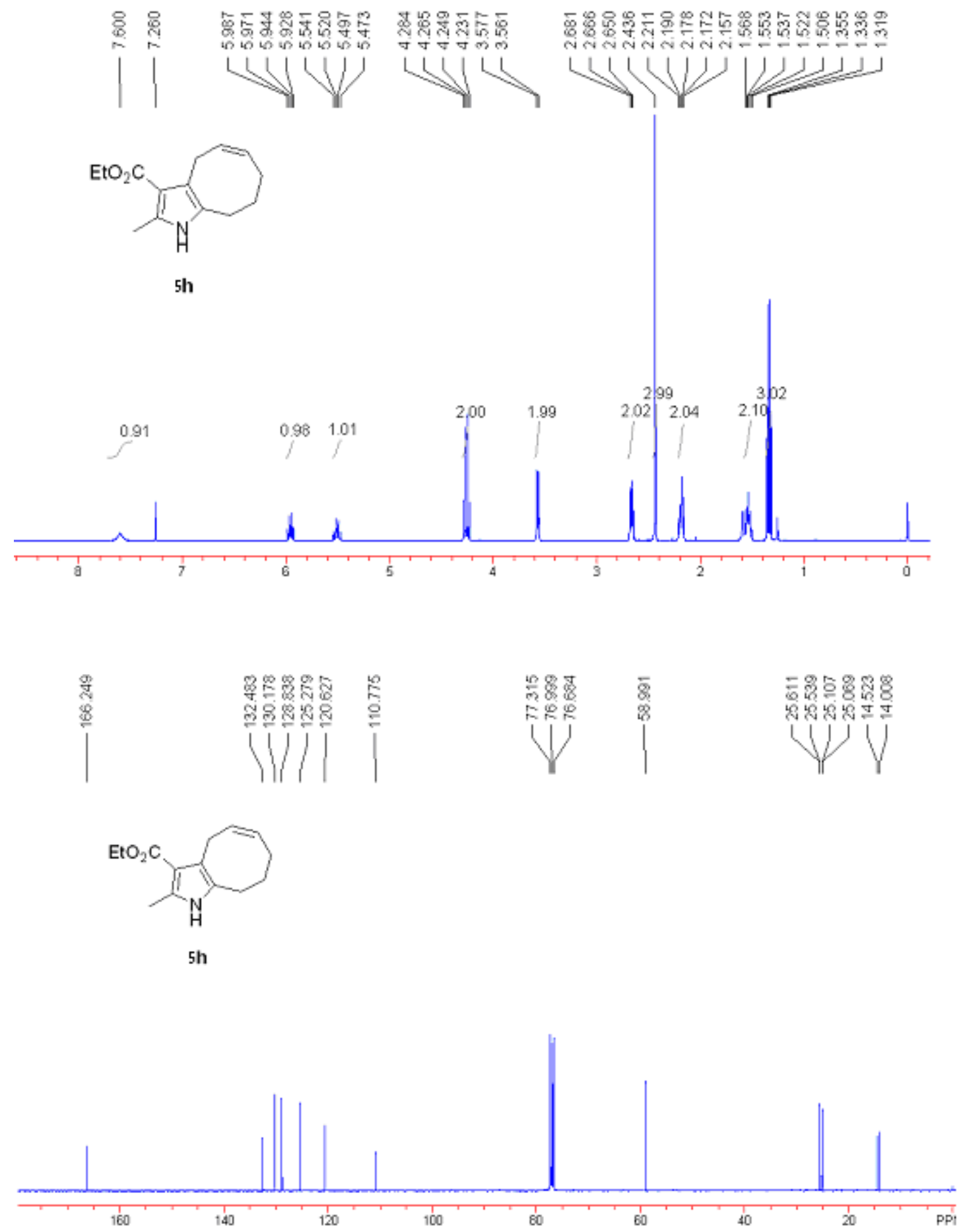




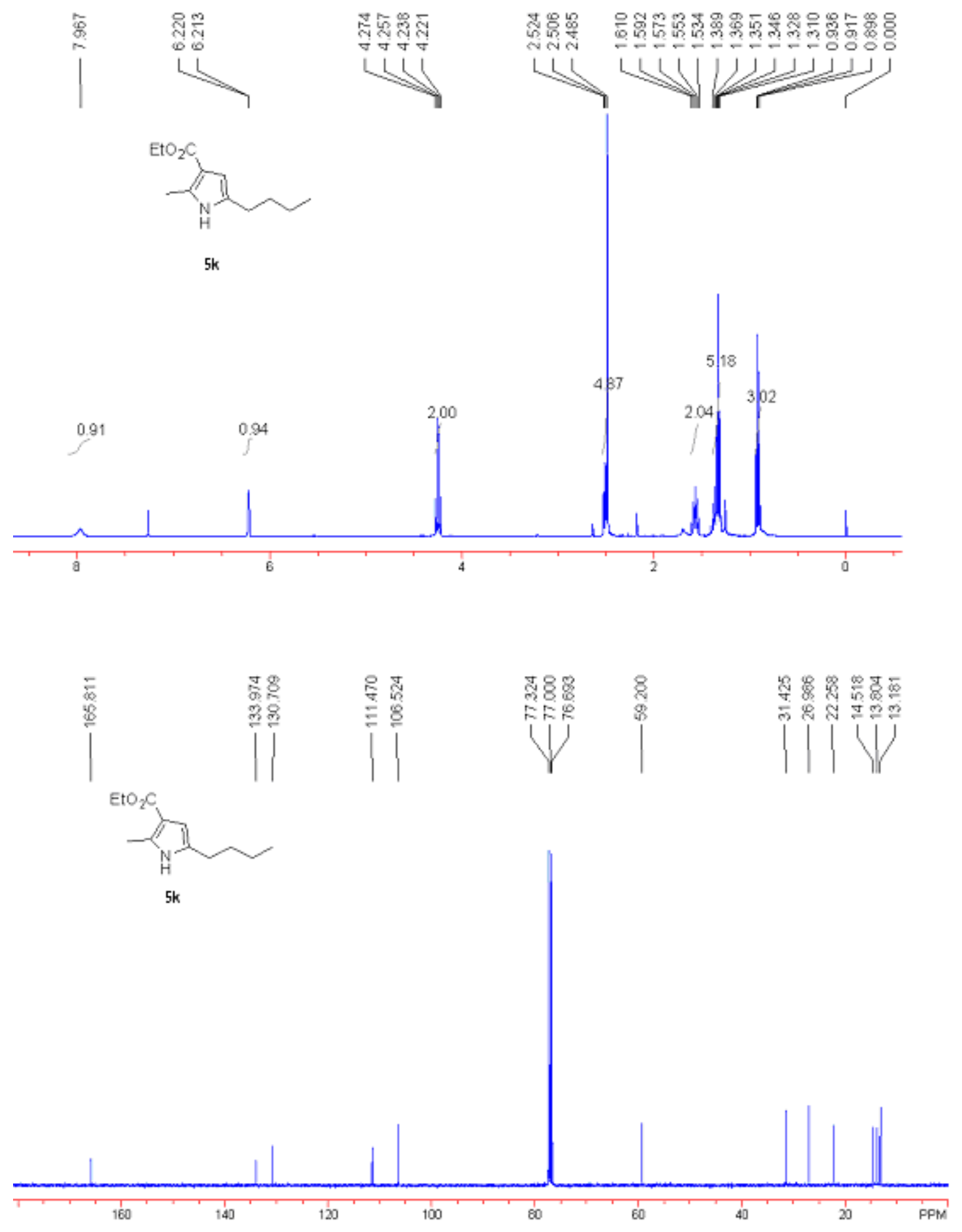




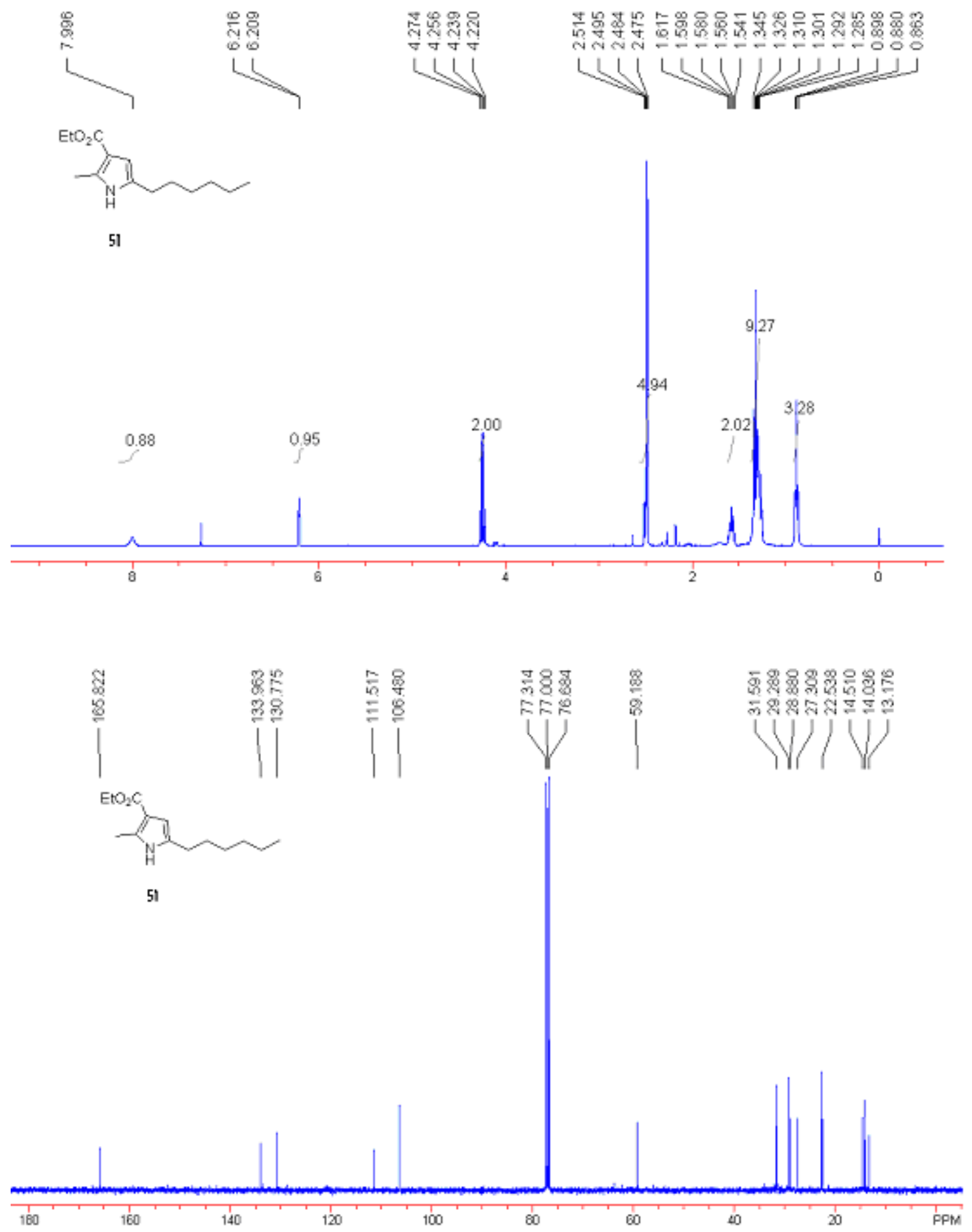




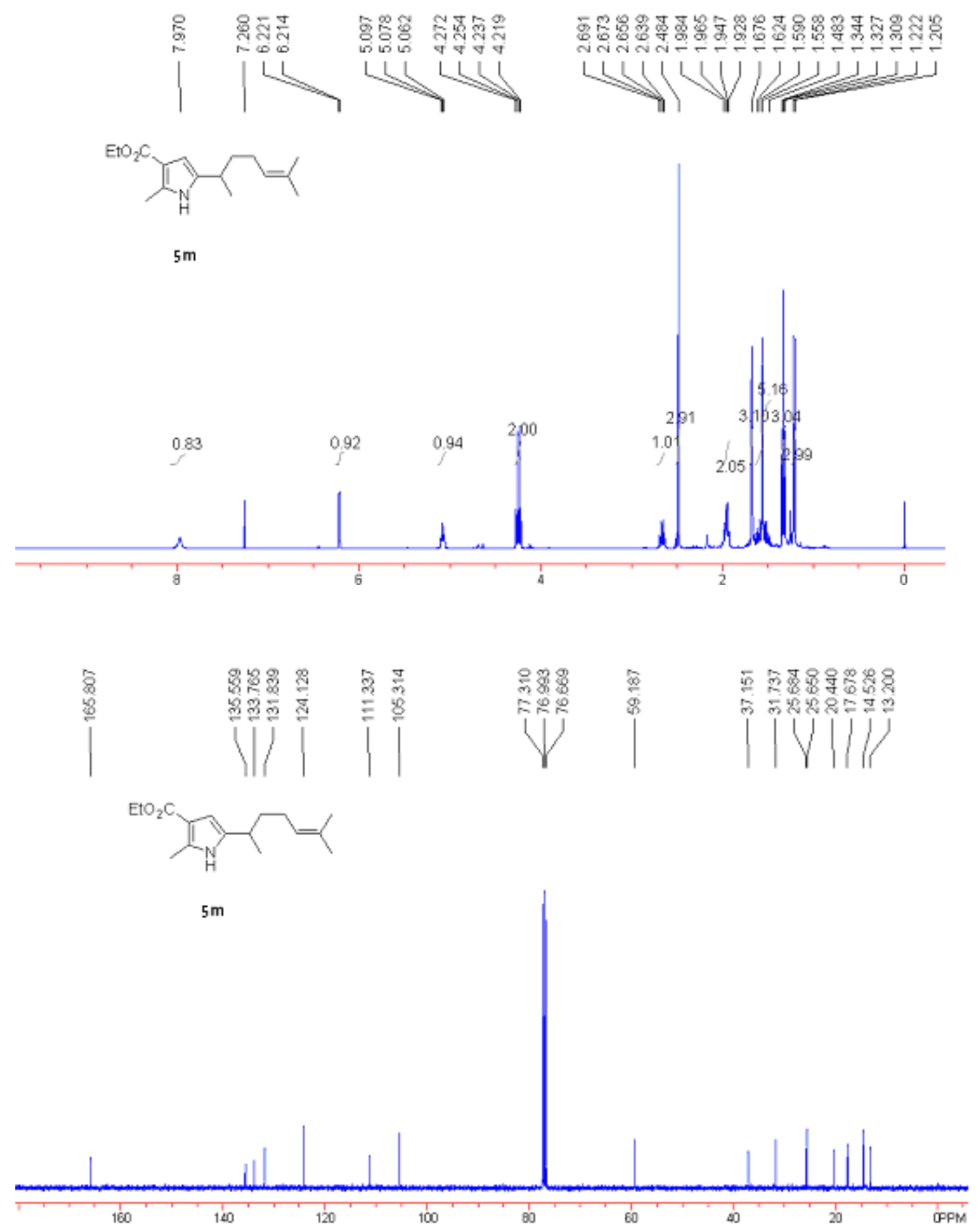


4. NOESY spectra for compounds $E-2 \mathrm{~d}$ and Z-3d

$E-2 d$

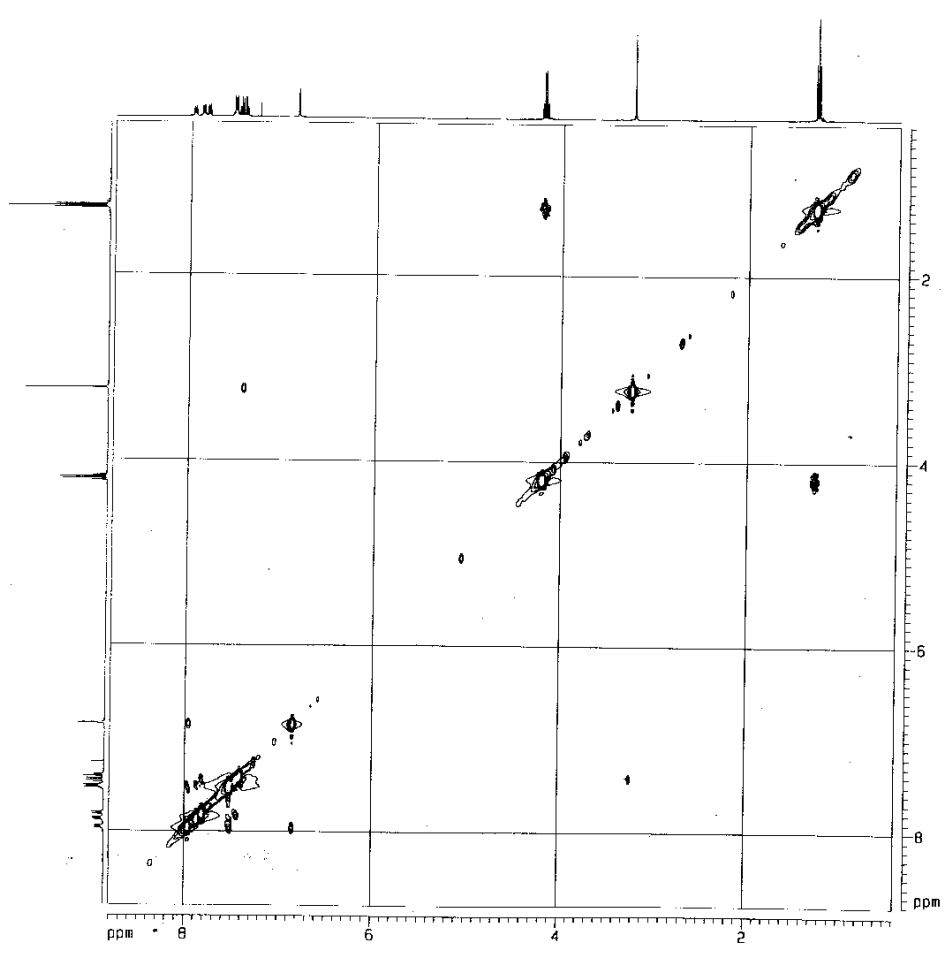

$5 r w-65$

$\underbrace{100}_{A_{1}}$

Z-3d 

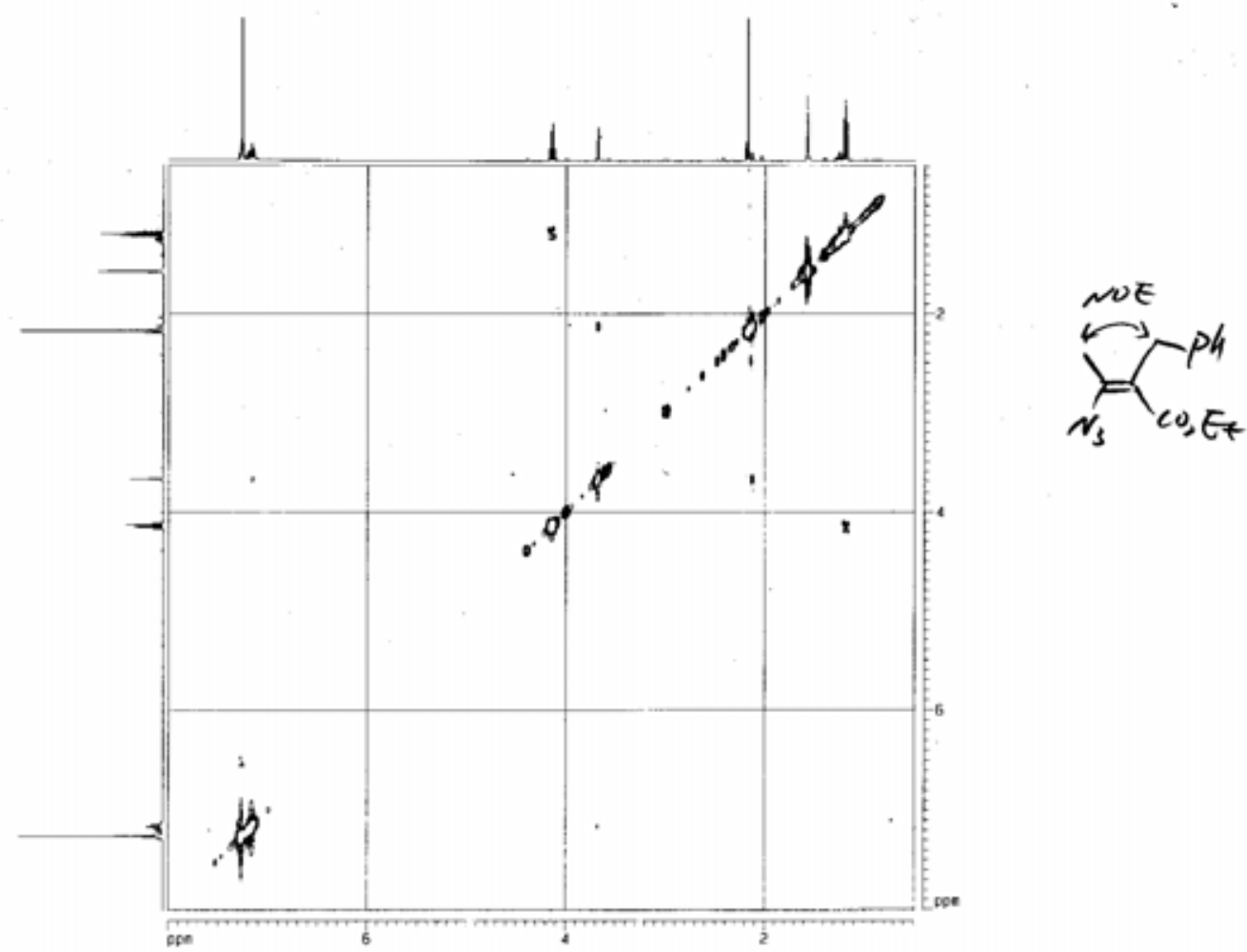

E-3d

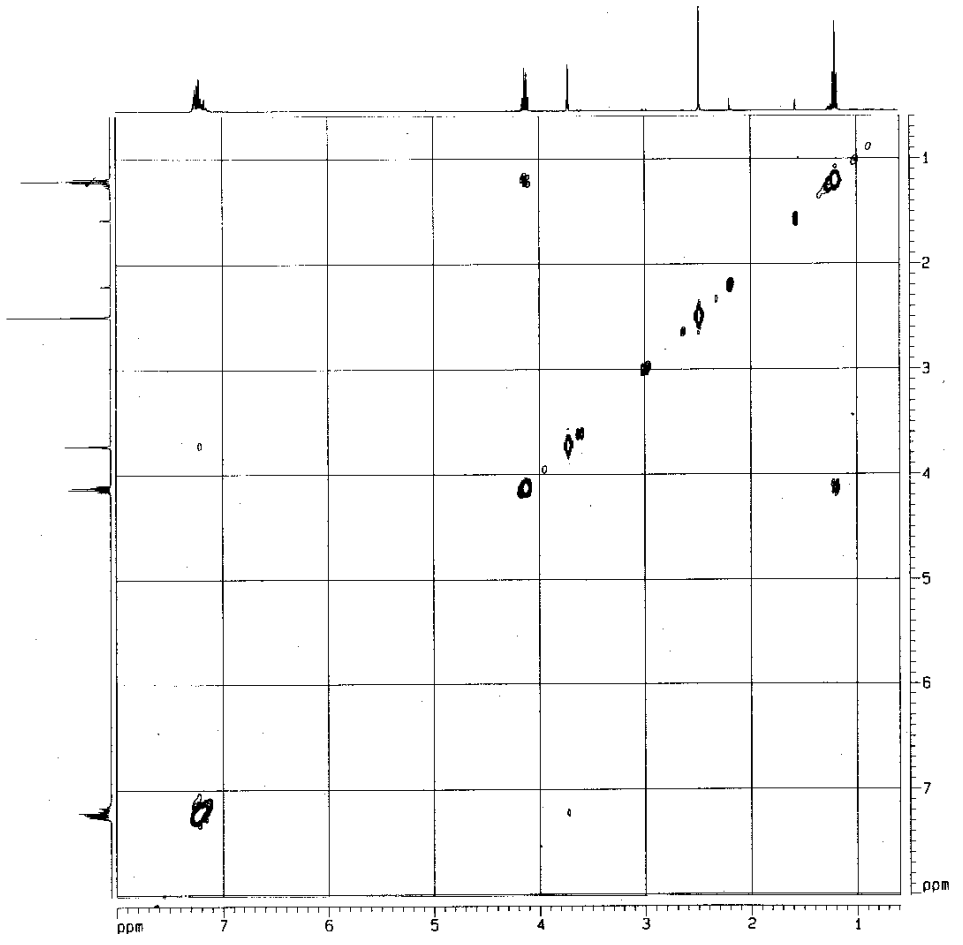

$5 r w-019$

${ }_{v_{3}}^{\text {or }}$ con

S41 